\title{
Rapidity and species dependence of particle production at large transverse momentum for $d+\mathrm{Au}$ collisions at $\sqrt{s_{\mathrm{NN}}}=200 \mathrm{GeV}$
}

B.I. Abelev, ${ }^{50}$ J. Adams, ${ }^{2}$ M.M. Aggarwal, ${ }^{30}$ Z. Ahammed, ${ }^{45}$ J. Amonett, ${ }^{20}$ B.D. Anderson, ${ }^{20}$ M. Anderson, ${ }^{6}$ D. Arkhipkin, ${ }^{13}$ G.S. Averichev,${ }^{12}$ Y. Bai,${ }^{28}$ J. Balewski,,${ }^{17}$ O. Barannikova, ${ }^{9}$ L.S. Barnby, ${ }^{2}$ J. Baudot, ${ }^{18}$ S. Bekele, ${ }^{29}$ V.V. Belaga, ${ }^{12}$ A. Bellingeri-Laurikainen, ${ }^{40}$ R. Bellwied, ${ }^{48}$ F. Benedosso, ${ }^{28}$

S. Bhardwaj, ${ }^{35}$ A. Bhasin, ${ }^{19}$ A.K. Bhati, ${ }^{30}$ H. Bichsel, ${ }^{47}$ J. Bielcik, ${ }^{50}$ J. Bielcikova, ${ }^{50}$ L.C. Bland,${ }^{3}$ S-L. Blyth, ${ }^{22}$ B.E. Bonner, ${ }^{36}$ M. Botje, ${ }^{28}$ J. Bouchet ${ }^{40}$ A.V. Brandin, ${ }^{26}$ A. Bravar, ${ }^{3}$ M. Bystersky, ${ }^{11}$ R.V. Cadman, ${ }^{1}$ X.Z. Cai ${ }^{39}$ H. Caines, ${ }^{50}$ M. Calderón de la Barca Sánchez, ${ }^{6}$ J. Castillo, ${ }^{28}$ O. Catu, ${ }^{50}$ D. Cebra,${ }^{6}$ Z. Chajecki, ${ }^{29}$ P. Chaloupka, ${ }^{11}$ S. Chattopadhyay ${ }^{45}$ H.F. Chen, ${ }^{38}$ J.H. Chen, ${ }^{39}$ J. Cheng, ${ }^{43}$

M. Cherney, ${ }^{10}$ A. Chikanian, ${ }^{50}$ W. Christie, ${ }^{3}$ J.P. Coffin, ${ }^{18}$ T.M. Cormier, ${ }^{48}$ M.R. Cosentino, ${ }^{37}$ J.G. Cramer, ${ }^{47}$ H.J. Crawford, ${ }^{5}$ D. Das ${ }^{45}$ S. Das, ${ }^{45}$ M. Daugherity ${ }^{42}$ M.M. de Moura, ${ }^{37}$ T.G. Dedovich, ${ }^{12}$ M. DePhillips, ${ }^{3}$ A.A. Derevschikov ${ }^{32}$ L. Didenko, ${ }^{3}$ T. Dietel,${ }^{14}$ P. Djawotho,,${ }^{17}$ S.M. Dogra, ${ }^{19}$ W.J. Dong, ${ }^{7}$ X. Dong, ${ }^{38}$ J.E. Draper, ${ }^{6}$ F. Du,${ }^{50}$ V.B. Dunin, ${ }^{12}$ J.C. Dunlop,${ }^{3}$ M.R. Dutta Mazumdar,${ }^{45}$ V. Eckardt, ${ }^{24}$ W.R. Edwards ${ }^{22}$ L.G. Efimov, ${ }^{12}$ V. Emelianov, ${ }^{26}$ J. Engelage,${ }^{5}$ G. Eppley,${ }^{36}$ B. Erazmus, ${ }^{40}$ M. Estienne,${ }^{18}$ P. Fachini,${ }^{3}$ R. Fatemi, ${ }^{23}$ J. Fedorisin, ${ }^{12}$ K. Filimonov, ${ }^{22}$ P. Filip, ${ }^{13}$ E. Finch, ${ }^{50}$ V. Fine, ${ }^{3}$ Y. Fisyak,${ }^{3}$ J. Fu, ${ }^{49}$ C.A. Gagliardi, ${ }^{41}$ L. Gaillard, ${ }^{2}$ M.S. Ganti, ${ }^{45}$ V. Ghazikhanian, ${ }^{7}$ P. Ghosh,${ }^{45}$ J.E. Gonzalez, ${ }^{7}$ Y.G. Gorbunov, ${ }^{10}$ H. Gos, ${ }^{46}$ O. Grebenyuk, ${ }^{28}$ D. Grosnick, ${ }^{44}$ S.M. Guertin, ${ }^{7}$ K.S.F.F. Guimaraes,${ }^{37}$ Y. Guo, ${ }^{48}$ N. Gupta, ${ }^{19}$ T.D. Gutierrez,${ }^{6}$ B. Haag, ${ }^{6}$ T.J. Hallman, ${ }^{3}$ A. Hamed, ${ }^{48}$ J.W. Harris, ${ }^{50}$ W. He, ${ }^{17}$ M. Heinz,${ }^{50}$ T.W. Henry, ${ }^{41}$ S. Hepplemann, ${ }^{31}$ B. Hippolyte, ${ }^{18}$ A. Hirsch, ${ }^{33}$ E. Hjort, ${ }^{22}$ A.M. Hoffman,${ }^{23}$ G.W. Hoffmann, ${ }^{42}$ M.J. Horner, ${ }^{22}$ H.Z. Huang, ${ }^{7}$ S.L. Huang, ${ }^{38}$ E.W. Hughes,${ }^{4}$ T.J. Humanic, ${ }^{29}$

G. Igo, ${ }^{7}$ P. Jacobs, ${ }^{22}$ W.W. Jacobs,${ }^{17}$ P. Jakl, ${ }^{11}$ F. Jia,${ }^{21}$ H. Jiang, ${ }^{7}$ P.G. Jones,${ }^{2}$ E.G. Judd, ${ }^{5}$

S. Kabana, ${ }^{40}$ K. Kang, ${ }^{43}$ J. Kapitan, ${ }^{11}$ M. Kaplan,${ }^{8}$ D. Keane,${ }^{20}$ A. Kechechyan, ${ }^{12}$ V.Yu. Khodyrev,${ }^{32}$

B.C. Kim ${ }^{34}$ J. Kiryluk, ${ }^{23}$ A. Kisiel,${ }^{46}$ E.M. Kislov, ${ }^{12}$ S.R. Klein, ${ }^{22}$ A. Kocoloski, ${ }^{23}$ D.D. Koetke, ${ }^{44}$

T. Kollegger, ${ }^{14}$ M. Kopytine, ${ }^{20}$ L. Kotchenda, ${ }^{26}$ V. Kouchpil, ${ }^{11}$ K.L. Kowalik, ${ }^{22}$ M. Kramer,${ }^{27}$

P. Kravtsov, ${ }^{26}$ V.I. Kravtsov, ${ }^{32}$ K. Krueger, ${ }^{1}$ C. Kuhn, ${ }^{18}$ A.I. Kulikov, ${ }^{12}$ A. Kumar, ${ }^{30}$ A.A. Kuznetsov, ${ }^{12}$

M.A.C. Lamont ${ }^{50}$ J.M. Landgraf, ${ }^{3}$ S. Lange, ${ }^{14}$ S. LaPointe,${ }^{48}$ F. Laue,${ }^{3}$ J. Lauret,${ }^{3}$ A. Lebedev ${ }^{3}$

R. Lednicky, ${ }^{13}$ C-H. Lee, ${ }^{34}$ S. Lehocka,${ }^{12}$ M.J. LeVine,${ }^{3}$ C. Li,${ }^{38}$ Q. Li, ${ }^{48}$ Y. Li,${ }^{43}$ G. Lin, ${ }^{50}$ X. Lin,,${ }^{49}$ S.J. Lindenbaum, ${ }^{27}$ M.A. Lisa, ${ }^{29}$ F. Liu ${ }^{49}$ H. Liu,${ }^{38}$ J. Liu,${ }^{36}$ L. Liu, ${ }^{49}$ Z. Liu, ${ }^{49}$ T. Ljubicic,${ }^{3}$ W.J. Llope,${ }^{36}$ H. Long, ${ }^{7}$ R.S. Longacre ${ }^{3}$ M. Lopez-Noriega,${ }^{29}$ W.A. Love, ${ }^{3}$ Y. Lu, ${ }^{49}$ T. Ludlam, ${ }^{3}$ D. Lynn, ${ }^{3}$ G.L. Ma, ${ }^{39}$ J.G. Ma, ${ }^{7}$ Y.G. Ma ${ }^{39}$ D. Magestro, ${ }^{29}$ D.P. Mahapatra,${ }^{15}$ R. Majka, ${ }^{50}$ L.K. Mangotra, ${ }^{19}$ R. Manweiler, ${ }^{44}$ S. Margetis, ${ }^{20}$ C. Markert, ${ }^{42}$ L. Martin,${ }^{40}$ H.S. Matis, ${ }^{22}$ Yu.A. Matulenko, ${ }^{32}$ C.J. McClain, ${ }^{1}$ T.S. McShane, ${ }^{10}$ Yu. Melnick, ${ }^{32}$ A. Meschanin, ${ }^{32}$ J. Millane, ${ }^{23}$ M.L. Miller, ${ }^{23}$ N.G. Minaev,${ }^{32}$ S. Mioduszewski, ${ }^{41}$ C. Mironov, ${ }^{20}$ A. Mischke, ${ }^{28}$ D.K. Mishra, ${ }^{15}$ J. Mitchell, ${ }^{36}$ B. Mohanty, ${ }^{22,45}$ L. Molnar, ${ }^{33}$ C.F. Moore, ${ }^{42}$ D.A. Morozov, ${ }^{32}$ M.G. Munhoz,${ }^{37}$ B.K. Nandi,,${ }^{16}$ C. Nattrass,${ }^{50}$ T.K. Nayak,${ }^{45}$ J.M. Nelson, ${ }^{2}$ P.K. Netrakanti, ${ }^{45}$ V.A. Nikitin, ${ }^{13}$ L.V. Nogach, ${ }^{32}$ S.B. Nurushev,${ }^{32}$ G. Odyniec, ${ }^{22}$ A. Ogawa,${ }^{3}$ V. Okorokov, ${ }^{26}$ M. Oldenburg, ${ }^{22}$ D. Olson, ${ }^{22}$ M. Pachr, ${ }^{11}$ S.K. Pal,${ }^{45}$ Y. Panebratsev,${ }^{12}$

S.Y. Panitkin, ${ }^{3}$ A.I. Pavlinov, ${ }^{48}$ T. Pawlak, ${ }^{46}$ T. Peitzmann, ${ }^{28}$ V. Perevoztchikov,${ }^{3}$ C. Perkins, ${ }^{5}$ W. Peryt,${ }^{46}$ V.A. Petrov, ${ }^{48}$ S.C. Phatak,${ }^{15}$ R. Picha,${ }^{6}$ M. Planinic,${ }^{51}$ J. Pluta, ${ }^{46}$ N. Poljak,${ }^{51}$ N. Porile ${ }^{33}$ J. Porter, ${ }^{47}$ A.M. Poskanzer, ${ }^{22}$ M. Potekhin,${ }^{3}$ E. Potrebenikova, ${ }^{12}$ B.V.K.S. Potukuchi, ${ }^{19}$ D. Prindle,${ }^{47}$ C. Pruneau, ${ }^{48}$ J. Putschke, ${ }^{22}$ G. Rakness, ${ }^{31}$ R. Raniwala, ${ }^{35}$ S. Raniwala, ${ }^{35}$ R.L. Ray, ${ }^{42}$ S.V. Razin, ${ }^{12}$ J. Reinnarth ${ }^{40}$ D. Relyea, ${ }^{4}$ F. Retiere, ${ }^{22}$ A. Ridiger, ${ }^{26}$ H.G. Ritter, ${ }^{22}$ J.B. Roberts, ${ }^{36}$ O.V. Rogachevskiy,${ }^{12}$ J.L. Romero, ${ }^{6}$ A. Rose,${ }^{22}$ C. Roy,${ }^{40}$ L. Ruan, ${ }^{22}$ M.J. Russcher, ${ }^{28}$ R. Sahoo, ${ }^{15}$ T. Sakuma, ${ }^{23}$ S. Salur, ${ }^{50}$

J. Sandweiss, ${ }^{50}$ M. Sarsour, ${ }^{41}$ P.S. Sazhin, ${ }^{12}$ J. Schambach, ${ }^{42}$ R.P. Scharenberg, ${ }^{33}$ N. Schmitz, ${ }^{24}$

K. Schweda, ${ }^{22}$ J. Seger, ${ }^{10}$ I. Selyuzhenkov ${ }^{48}$ P. Seyboth, ${ }^{24}$ A. Shabetai, ${ }^{22}$ E. Shahaliev, ${ }^{12}$ M. Shao,${ }^{38}$ M. Sharma, ${ }^{30}$ W.Q. Shen, ${ }^{39}$ S.S. Shimanskiy, ${ }^{12}$ E Sichtermann, ${ }^{22}$ F. Simon, ${ }^{23}$ R.N. Singaraju, ${ }^{45}$

N. Smirnov, ${ }^{50}$ R. Snellings, ${ }^{28}$ G. Sood,${ }^{44}$ P. Sorensen,${ }^{3}$ J. Sowinski, ${ }^{17}$ J. Speltz, ${ }^{18}$ H.M. Spinka,,${ }^{1}$ B. Srivastava, ${ }^{33}$ A. Stadnik, ${ }^{12}$ T.D.S. Stanislaus, ${ }^{44}$ R. Stock, ${ }^{14}$ A. Stolpovsky,${ }^{48}$ M. Strikhanov, ${ }^{26}$ B. Stringfellow, ${ }^{33}$ A.A.P. Suaide, ${ }^{37}$ E. Sugarbaker,${ }^{29}$ M. Sumbera, ${ }^{11}$ Z. Sun,${ }^{21}$ B. Surrow,${ }^{23}$ M. Swanger, ${ }^{10}$ T.J.M. Symons, ${ }^{22}$ A. Szanto de Toledo, ${ }^{37}$ A. Tai, ${ }^{7}$ J. Takahashi, ${ }^{37}$ A.H. Tang, ${ }^{3}$ T. Tarnowsky, ${ }^{33}$ D. Thein, ${ }^{7}$

J.H. Thomas, ${ }^{22}$ A.R. Timmins, ${ }^{2}$ S. Timoshenko, ${ }^{26}$ M. Tokarev,${ }^{12}$ T.A. Trainor, ${ }^{47}$ S. Trentalange,${ }^{7}$ R.E. Tribble, ${ }^{41}$ O.D. Tsai, ${ }^{7}$ J. Ulery ${ }^{33}$ T. Ullrich, ${ }^{3}$ D.G. Underwood, ${ }^{1}$ G. Van Buren,${ }^{3}$ N. van der Kolk,${ }^{28}$ M. van Leeuwen, ${ }^{22}$ A.M. Vander Molen, ${ }^{25}$ R. Varma,${ }^{16}$ I.M. Vasilevski, ${ }^{13}$ A.N. Vasiliev,${ }^{32}$ R. Vernet,${ }^{18}$ 
S.E. Vigdor, ${ }^{17}$ Y.P. Viyogi, ${ }^{15}$ S. Vokal, ${ }^{12}$ S.A. Voloshin, ${ }^{48}$ W.T. Waggoner, ${ }^{10}$ F. Wang, ${ }^{33}$ G. Wang, ${ }^{7}$ J.S. Wang, ${ }^{21}$ X.L. Wang, ${ }^{38}$ Y. Wang, ${ }^{43}$ J.W. Watson, ${ }^{20}$ J.C. Webb, ${ }^{44}$ G.D. Westfall, ${ }^{25}$ A. Wetzler, ${ }^{22}$ C. Whitten Jr., ${ }^{7}$ H. Wieman, ${ }^{22}$ S.W. Wissink, ${ }^{17}$ R. Witt, ${ }^{50}$ J. Wood, ${ }^{7}$ J. Wu, ${ }^{38}$ N. Xu, ${ }^{22}$ Q.H. Xu, ${ }^{22}$ Z. Xu, ${ }^{3}$ P. Yepes, ${ }^{36}$ I-K. Yoo, ${ }^{34}$ V.I. Yurevich, ${ }^{12}$ W. Zhan, ${ }^{21}$ H. Zhang, ${ }^{3}$ W.M. Zhang, ${ }^{20}$ Y. Zhang, ${ }^{38}$ Z.P. Zhang, ${ }^{38}$ Y. Zhao, ${ }^{38}$ C. Zhong, ${ }^{39}$ R. Zoulkarneev, ${ }^{13}$ Y. Zoulkarneeva, ${ }^{13}$ A.N. Zubarev, ${ }^{12}$ and J.X. Zuo ${ }^{39}$ (STAR Collaboration)

\author{
${ }^{1}$ Argonne National Laboratory, Argonne, Illinois 60439 \\ ${ }^{2}$ University of Birmingham, Birmingham, United Kingdom \\ ${ }^{3}$ Brookhaven National Laboratory, Upton, New York 11973 \\ ${ }^{4}$ California Institute of Technology, Pasadena, California 91125 \\ ${ }^{5}$ University of California, Berkeley, California 94720 \\ ${ }^{6}$ University of California, Davis, California 95616 \\ ${ }^{7}$ University of California, Los Angeles, California 90095 \\ ${ }^{8}$ Carnegie Mellon University, Pittsburgh, Pennsylvania 15213 \\ ${ }^{9}$ University of Illinois, Chicago \\ ${ }^{10}$ Creighton University, Omaha, Nebraska 68178 \\ ${ }^{11}$ Nuclear Physics Institute AS CR, 25068 Řež/Prague, Czech Republic \\ ${ }^{12}$ Laboratory for High Energy (JINR), Dubna, Russia \\ ${ }^{13}$ Particle Physics Laboratory (JINR), Dubna, Russia \\ ${ }^{14}$ University of Frankfurt, Frankfurt, Germany \\ ${ }^{15}$ Institute of Physics, Bhubaneswar 751005, India \\ ${ }^{16}$ Indian Institute of Technology, Mumbai, India \\ ${ }^{17}$ Indiana University, Bloomington, Indiana 47408 \\ ${ }^{18}$ Institut de Recherches Subatomiques, Strasbourg, France \\ ${ }^{19}$ University of Jammu, Jammu 180001, India \\ ${ }^{20}$ Kent State University, Kent, Ohio 44242 \\ ${ }^{21}$ Institute of Modern Physics, Lanzhou, China \\ ${ }^{22}$ Lawrence Berkeley National Laboratory, Berkeley, California 94720 \\ ${ }^{23}$ Massachusetts Institute of Technology, Cambridge, MA 02139-4307 \\ ${ }^{24}$ Max-Planck-Institut für Physik, Munich, Germany \\ ${ }^{25}$ Michigan State University, East Lansing, Michigan 48824 \\ ${ }^{26}$ Moscow Engineering Physics Institute, Moscow Russia \\ ${ }^{27}$ City College of New York, New York City, New York 10031 \\ ${ }^{28}$ NIKHEF and Utrecht University, Amsterdam, The Netherlands \\ ${ }^{29}$ Ohio State University, Columbus, Ohio 43210 \\ ${ }^{30}$ Panjab University, Chandigarh 160014, India \\ ${ }^{31}$ Pennsylvania State University, University Park, Pennsylvania 16802 \\ ${ }^{32}$ Institute of High Energy Physics, Protvino, Russia \\ ${ }^{33}$ Purdue University, West Lafayette, Indiana 47907 \\ ${ }^{34}$ Pusan National University, Pusan, Republic of Korea \\ ${ }^{35}$ University of Rajasthan, Jaipur 302004, India \\ ${ }^{36}$ Rice University, Houston, Texas 77251 \\ ${ }^{37}$ Universidade de Sao Paulo, Sao Paulo, Brazil \\ ${ }^{38}$ University of Science \&5 Technology of China, Hefei 230026, China \\ ${ }^{39}$ Shanghai Institute of Applied Physics, Shanghai 201800, China \\ ${ }^{40}$ SUBATECH, Nantes, France \\ ${ }^{41}$ Texas A\&M University, College Station, Texas 77843 \\ ${ }^{42}$ University of Texas, Austin, Texas 78712 \\ ${ }^{43}$ Tsinghua University, Beijing 100084, China \\ ${ }^{44}$ Valparaiso University, Valparaiso, Indiana 46383 \\ ${ }^{45}$ Variable Energy Cyclotron Centre, Kolkata 700064, India \\ ${ }^{46}$ Warsaw University of Technology, Warsaw, Poland \\ ${ }^{47}$ University of Washington, Seattle, Washington 98195 \\ ${ }^{48}$ Wayne State University, Detroit, Michigan 48201 \\ ${ }^{49}$ Institute of Particle Physics, CCNU (HZNU), Wuhan 430079, China \\ ${ }^{50}$ Yale University, New Haven, Connecticut 06520 \\ ${ }^{51}$ University of Zagreb, Zagreb, HR-10002, Croatia
}

(Dated: December 18, 2006)

We determine rapidity asymmetry in the production of charged pions, protons and anti-protons for large transverse momentum $\left(p_{\mathrm{T}}\right)$ for $d+\mathrm{Au}$ collisions at $\sqrt{s_{\mathrm{NN}}}=200 \mathrm{GeV}$. The rapidity asymmetry 
is defined as the ratio of particle yields at backward rapidity (Au beam direction or -ve rapidity) to those at forward rapidity ( $d$ beam direction or +ve rapidity). The identified hadrons are measured in the rapidity regions $|y|<0.5$ and $0.5<|y|<1.0$ for the $p_{\mathrm{T}}$ range $2.5<p_{\mathrm{T}}<10 \mathrm{GeV} / c$. We observe significant rapidity asymmetry for charged pion and proton+anti-proton production in both rapidity regions. The asymmetry is larger for $0.5<|y|<1.0$ than for $|y|<0.5$ and is almost independent of particle type. The measurements are compared to various model predictions employing multiple scattering, energy loss, nuclear shadowing, saturation effects, and recombination, and also to a phenomenological parton model. We find that asymmetries are sensitive to model parameters and show model-preference. The rapidity dependence of $\pi^{-} / \pi^{+}$and $\bar{p} / p$ ratios in peripheral $d+\mathrm{Au}$ and forward neutron-tagged events are used to study the contributions of valence quarks and gluons to particle production at high $p_{\mathrm{T}}$. The results are compared to calculations based on NLO pQCD and other measurements of quark fragmentation functions.

PACS numbers: 25.75.-q,25.75.Dw,13.85.-t

\section{INTRODUCTION}

The mechanisms for particle production in $d+\mathrm{Au}$ collisions at RHIC may be different at forward and backward rapidities. The partons from the deuteron-side (forward rapidity) are expected to undergo multiple scattering while traversing the gold nucleus. Those on the gold-side (backward rapidity) are likely to be affected by the properties of the nucleus. A comparative study of particle production at forward and backward rapidity can be carried out using a ratio called the rapidity asymmetry $\left(Y_{\text {Asym }}\right)$, which is defined as,

$$
Y_{\text {Asym }}\left(p_{\mathrm{T}}\right)=\frac{Y_{\mathrm{B}}\left(p_{\mathrm{T}}\right)}{Y_{\mathrm{F}}\left(p_{\mathrm{T}}\right)},
$$

where $Y_{\mathrm{F}}$ and $Y_{\mathrm{B}}$ are forward and backward particle yields, respectively. $Y_{\text {Asym }}$ may provide unique information to help determine the relative contributions of various physics processes to particle production, such as multiple scattering, nuclear shadowing, recombination of thermal partons, and parton saturation.

Recently, models incorporating different physics effects have described the nuclear modification factor for $d+\mathrm{Au}$ collisions $\left(R_{\mathrm{dAu}}\right)$. Models including shadowing effects or nuclear modifications to the nucleon parton distributions reproduce reasonably well $R_{\mathrm{dAu}}$ for inclusive charged hadrons [1]. Those based on transverse momentum broadening (Cronin effect [2]), dynamical shadowing, and energy loss in cold nuclear matter [3], also give $R_{\mathrm{dAu}}$ predictions for inclusive charged hadrons, consistent with experimental data. Models based on the color glass condensate (CGC) approach reproduce the $p_{\mathrm{T}}$ dependence of inclusive charged hadron $R_{\mathrm{dAu}}$ at both mid- and forward-rapidity [4]. These models also qualitatively describe the pseudorapidity asymmetry for inclusive charged hadrons in $d+\mathrm{Au}$ collisions [5].

Another approach based on hadronization by recombination of thermal partons at lower $p_{\mathrm{T}}$ has been quite successful in describing the observed $R_{\mathrm{dAu}}$ for charged hadrons at RHIC [6]. This approach emphasizes the hadronization portion of the final state interaction. Although it takes into account the hard scattering in pQCD, the fragmentation is replaced by recombination of soft and shower partons in the intermediate $p_{\mathrm{T}}$ region. Also, a phenomenological approach, called EPOS [7], based on a parton model, has described the $d+\mathrm{Au}$ collision data at RHIC. In this model the nuclear effects are included through elastic and inelastic parton ladder splitting.

It is of interest to see how these models compare to data for rapidity asymmetry of identified hadrons from $d+\mathrm{Au}$ collisions. More precisely, identified hadron $Y_{\text {Asym }}$, a more differential quantity, may allow some determination of the relative contribution of the physical processes discussed above. Strong particle type (baryon and meson) dependence of the nuclear modification factor and azimuthal anisotropy at intermediate $p_{\mathrm{T}}\left(2<p_{\mathrm{T}}<6 \mathrm{GeV} / c\right)$ has been observed in $\mathrm{Au}+\mathrm{Au}$ collisions at RHIC [8]. The present study will investigate if such particle type (baryon and meson) dependence is observed in $Y_{\text {Asym }}$ for $d+\mathrm{Au}$ collisions.

In addition to providing insight into different particle production mechanisms at forward and backward rapidity for $d+\mathrm{Au}$ collisions, the measurements presented here may be used to study the presence of possible effects of valence quarks and isospin on particle production. At high $p_{\mathrm{T}}$ and rapidities away from midrapidity, the role of valence quarks becomes increasingly dominant. Such studies are even more interesting for $n$-tag events (events where the neutron in the deuteron does not interact with the gold nucleus). A comparative study between $p+\mathrm{Au}(n-$ $\operatorname{tag})$ and $d+\mathrm{Au}$ data is of interest. For $n$-tag events at forward rapidity and high $p_{\mathrm{T}}$, the two valence $u$ quarks in the proton of the deuteron should lead to more production of $\pi^{+}(u \bar{d})$ compared to $\pi^{-}(d \bar{u})$. For backward rapidities, if the flavor distribution in sea quarks is uniform and the incoming gold nucleus has no asymmetry in $u$ and $d$ quarks, one expects the ratio $\pi^{-} / \pi^{+} \sim 1$. This difference between forward and backward rapidity may be more pronounced for 
$\bar{p} / p$. Study of particle ratios as a function of rapidity at high $p_{\mathrm{T}}$ in peripheral and $n$-tag events for $d+\mathrm{Au}$ collisions may provide some information on the flavor dependence of particle production. These ratios are in principle sensitive to the fragmentation function ratios of $u$-quarks to $\pi^{-}$and $\pi^{+}$[9], to the ratio of $(u, d)$-quarks fragmenting to protons [10], and to the fractional contributions of quarks and gluons to hadrons at the given momentum.

In this paper, we present the first results for the rapidity asymmetry of charged pion, proton and anti-proton production at high $p_{\mathrm{T}}$ for $d+\mathrm{Au}$ collisions at $\sqrt{s_{\mathrm{NN}}}=200 \mathrm{GeV}$ measured by the STAR experiment [11] at RHIC. A similar study for inclusive charged hadrons has been reported in Ref. [5]. The asymmetry is studied as a function of $p_{\mathrm{T}}$ for different collision centralities in the two rapidity regions $|y|<0.5$ and $0.5<|y|<1.0$. In section II we discuss the detectors used in the analysis, trigger and centrality selection, particle identification at high $p_{\mathrm{T}}$, and the systematic errors. In section III we discuss the rapidity, $p_{\mathrm{T}}$, species, and centrality dependence of $Y_{\text {Asym }}$. In section IV, the $Y_{\text {Asym }}$ results are compared to calculations from various models discussed earlier. In section $\mathrm{V}$, we present the rapidity dependence of the nuclear modification factor for $\pi^{+}+\pi^{-}$and $p+\bar{p}$. In section VI, we study the anti-particle to particle ratios as a function of rapidity at high $p_{\mathrm{T}}$ in $n$-tag and peripheral $d+\mathrm{Au}$ events in order to investigate the flavor dependence of particle production. Section VII completes this work with the summary of our findings.

\section{EXPERIMENT AND ANALYSIS}

\section{A. Detectors}

For the present analysis we use data recorded by the Time Projection Chamber (TPC) [12] in the STAR experiment at RHIC. The TPC is STAR's primary tracking device. It is $4.2 \mathrm{~m}$ long and $4 \mathrm{~m}$ in diameter. The sensitive volume of the TPC contains P10 gas (10\% methane, 90\% argon) regulated at 2 mbar above atmospheric pressure. The TPC data are used to determine particle trajectories, momenta, and particle-type through ionization energy loss $(d E / d x)$. Its acceptance covers \pm 1.8 units of pseudorapidity $(\eta)$ and the full azimuthal angle. Charged particle momenta are determined from the TPC data for the $d+\mathrm{Au}$ run in the year 2003 in which STAR's solenoidal magnet field was set to 0.5 T. Two Zero Degree Calorimeters (ZDCs) [13] situated along both sides of the beam axis, about $18 \mathrm{~m}$ from the nominal collision point (center of TPC), were used for triggering. The collision centrality is obtained from the charged hadron multi-
TABLE I: Centrality selection, number of participating nucleons, and number of binary collisions for $d+\mathrm{Au}$ collisions at $\sqrt{s_{\mathrm{NN}}}=200 \mathrm{GeV}$.

\begin{tabular}{cccc}
\hline \% cross section & $N_{\text {chtrk }}^{\mathrm{FTPC}}$ & $\left\langle N_{\text {part }}\right\rangle$ & $\left\langle N_{\text {bin }}\right\rangle$ \\
\hline $0-20$ & $>17$ & $15.67 \pm 1.07$ & $15.1 \pm 1.15$ \\
$20-40$ & $11-17$ & $11.16 \pm 1.25$ & $10.6 \pm 1.38$ \\
$40-100$ & $<11$ & $5.14 \pm 0.47$ & $4.2 \pm 0.51$ \\
$0-100$ & $>0$ & $8.31 \pm 0.34$ & $7.5 \pm 0.38$ \\
\hline
\end{tabular}

plicity measured by STAR's Forward Time Projection Chambers (FTPCs) [14]. The details of the design and other characteristics of the detectors can be found in Ref. [11]. The details of the trigger condition, collision centrality selection, and method of high $p_{\mathrm{T}}$ particle identification are described below.

\section{B. Trigger conditions}

The ZDC in the Au beam direction, which is assigned negative pseudorapidity $(\eta)$, was used as the trigger detector for obtaining the minimum bias data. The minimum bias trigger required at least one beam-rapidity neutron in the ZDC. The trigger efficiency was found to be $95 \pm 3 \%$ of the $d+\mathrm{Au}$ hadronic cross section $\sigma_{h a d r}^{d A u}$. Trigger backgrounds were determined using data recorded for beam crossings without collisions. For the $n$-tag events, the ZDC in the deuteron beam direction was used. Such events were required to have at least one beam rapidity neutron in the ZDC. The cross section for such a process was measured to be $(19.2 \pm 1.3) \%$ of $\sigma_{h a d r}^{d A u}$. The vertex was reconstructed for $93 \pm 1 \%$ of triggered minimum bias events. A total of 11.7 million minimum bias $d+\mathrm{Au}$ events and 2.0 million $n$ tag events having a vertex within $\pm 30 \mathrm{~cm}$ of the nominal interaction point along the beam direction were analyzed. Two rapidity regions were used: $|y|<0.5$ and $0.5<|y|<1.0$, and the $p_{\mathrm{T}}$ range was $2.5<p_{\mathrm{T}}<10 \mathrm{GeV} / c$. The $p_{\mathrm{T}}$ spectra were corrected for trigger and vertex-finding inefficiencies. Further details of trigger conditions for the minimum bias data can be found in Ref. [15].

\section{Collision centrality}

Uncorrected charged track multiplicity $\left(N_{\text {chtrk }}^{\mathrm{FTPC}}\right)$ measured within $-3.8<\eta<-2.8$ by the FTPC was used to determine the collision centrality for $d+\mathrm{Au}$ collisions. Figure 1 shows the charged track multiplicity in FTPC in the Au beam direction for minimum bias $d+\mathrm{Au}$ collisions and ZDC neutron-tagged events. The latter have a strong bias toward low multiplicity. The FTPC in the Au beam direction and the ZDC in $d$ beam direction are separated by 


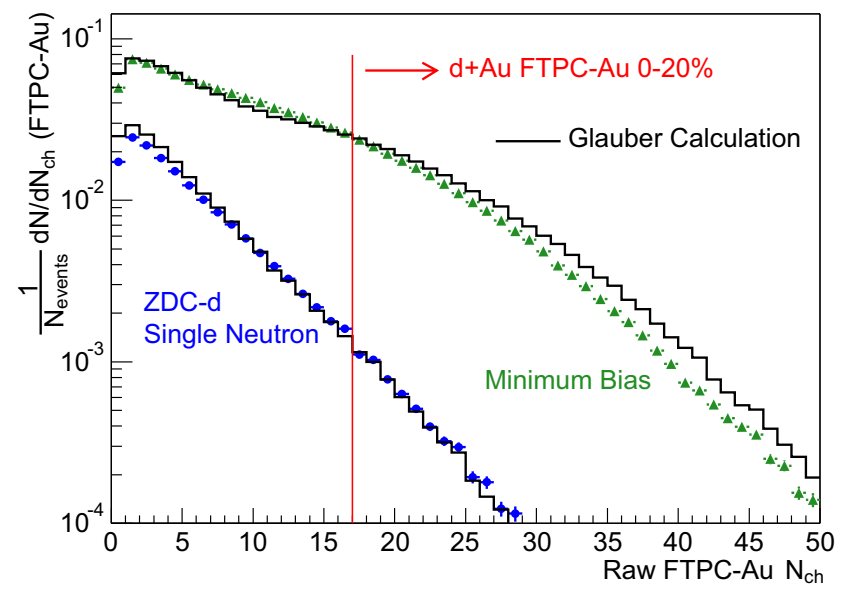

FIG. 1: (Color online) Uncorrected charged particle multiplicity distributions measured in $-3.8<\eta<-2.8$ (Audirection) for $\mathrm{d}+\mathrm{Au}$ collisions. Points are for minimum bias (triangles) and peripheral (circles, ZDC-d single neutron) collisions [15]. Both are normalized to the total number of $d+A u$ collisions. Histograms are Glauber model calculations.

8 units in rapidity. The centrality selection criteria is given in Table I, along with the average number of binary collisions $\left(N_{\text {bin }}\right)$ and the number of participating nucleons $\left(N_{\text {part }}\right)$ estimated using a Monte Carlo Glauber calculation [16] incorporating the Hulthén wave function of the deuteron [17]. In this model $\sigma_{\text {hadr }}^{d A u}=2.21 \pm 0.09 \mathrm{~b}$, and $N_{\mathrm{bin}}$ for $n$-tag events is $2.9 \pm 0.2$. The FTPC (Au beam direction) multiplicity distribution was modeled by convoluting the Glauber model distribution of participants from the $\mathrm{Au}$ nucleus with the charged multiplicity distribution measured in $2.5<|\eta|<3.5$ for $\bar{p}+p$ collisions at $\sqrt{s}=200 \mathrm{GeV}$ [18]. The FTPC acceptance, efficiency and backgrounds were taken into account using HIJING [19] events in a GEANT model of the detector. This model provides reasonable agreement with the measured charged track multiplicity distribution in the FTPC and the single neutron cross section measured by the ZDC on the deuteron side (Fig. 1). In Fig. 1 we also show the cut defining the $20 \%$ highest multiplicity collisions in the $d+\mathrm{Au}$ data. Further details of centrality tagging in $d+\mathrm{Au}$ collisions can be found in Ref. [15].

\section{Particle identification at high $p_{\mathrm{T}}$}

Particle identification at high transverse momenta $\left(p_{\mathrm{T}}>2.5 \mathrm{GeV} / c\right)$ is done by exploiting the relativistic rise of the ionization energy loss. Here we briefly describe the identification procedure (see Ref. [20, 21]). For $2.5<p_{T} \lesssim 10 \mathrm{GeV} / c$, there is a

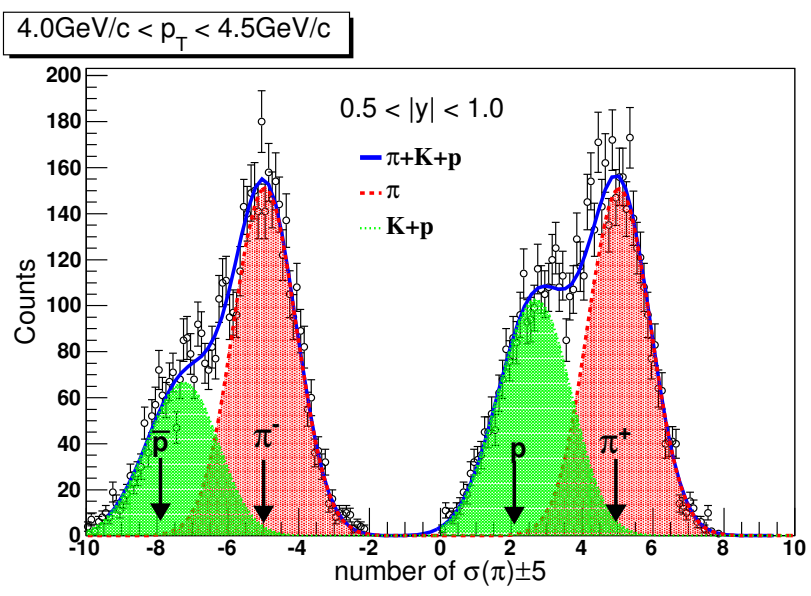

FIG. 2: (Color online) $d E / d x$ distribution normalized by pion $d E / d x$ at $4.0<p_{\mathrm{T}}<4.5 \mathrm{GeV} / c$ and $0.5<|\eta|<1.0$, shifted by \pm 5 for positive and negative charged particles, respectively. The distributions are for minimum bias $d+\mathrm{Au}$ collisions. The pion, proton, and anti-proton peak positions are indicated by arrows.

difference of about $10-20 \%$ between the pion $d E / d x$ and the $d E / d x$ for kaons and protons, due to the relativistic rise of the ionization energy loss for pions. This results in a few sigma (1-3 $\sigma)$ separation. The $d E / d x$ resolution is $\sim 8 \%[20]$.

Pions are the dominant component of the hadron yield for $d+\mathrm{Au}$ collisions at RHIC. The prominent peak in the $d E / d x$ distribution is used to determine the pion yield in this $p_{\mathrm{T}}$ range. To extract the pion yield in a given $p_{\mathrm{T}}$ bin, we performed a six Gaussian fit to the normalized $d E / d x$ distributions of positive and negative hadrons simultaneously. The normalized $d E / d x$ in general is defined as $n \sigma_{X}^{Y}=\log \left((d E / d x)_{Y} / B_{X}\right) / \sigma_{X}$, where $X, Y$ can be $e^{ \pm}, \pi^{ \pm}, K^{ \pm}$or $p(\bar{p})$. $B_{X}$ is the expected mean $d E / d x$ of particle $X$, and $\sigma_{X}$ is the $d E / d x$ resolution of the TPC and is a function of the track length in TPC. The expected mean $d E / d x$ of particle $X$ is calculated using Bichsel function for the energy loss in thin layers of P10 for STAR TPC $[12,22]$. The good agreement between the measurement and the calculation can be found in Ref. [20].

Fig. 2 shows a typical $d E / d x$ distribution normalized to pion $d E / d x$ (referred to as the $n \sigma_{\pi}$ distribution) for charged hadrons with $4.0<p_{\mathrm{T}}<4.5 \mathrm{GeV} / c$ and $0.5<|\eta|<1.0$. For clarity of presentation, the $n \sigma_{\pi}$ distributions in Fig. 2 are shifted by \pm 5 for positive and negative charged particles, respectively. The $n \sigma_{\pi}^{\pi}$ distribution is a normal Gaussian distribution with an ideal calibration. The six Gaussians are for $\pi^{ \pm}, K^{ \pm}$and $p(\bar{p})$. The relative peak positions of the kaons $\left(n \sigma_{\pi}^{K}-n \sigma_{\pi}^{\pi}\right)$ and protons $\left(n \sigma_{\pi}^{p(\bar{p})}-n \sigma_{\pi}^{\pi}\right)$ with respect to pion peak position in the $n \sigma_{\pi}$ distri- 
bution are estimated by studying the difference between $d E / d x$ distribution normalized to pion $d E / d x$ and $d E / d x$ distribution normalized to kaon $d E / d x$ $\left(n \sigma_{\pi}^{\pi}-n \sigma_{K}^{K}\right), d E / d x$ distribution normalized to proton $d E / d x\left(n \sigma_{\pi}^{\pi}-n \sigma_{p}^{p}\right)$ and for positively and negatively charged particles $\left(n \sigma_{\pi}^{h^{+}}-n \sigma_{\pi}^{h^{-}}\right)$. The widths of the six Gaussians are taken to be the same. The Gaussian distribution used to extract the pion yield and the pion, proton and anti-proton peak positions are also shown in the figure.

The proton yield is obtained by integrating the entries $(Y)$ in the low part of the $d E / d x$ distribution, about $2.5 \sigma$ away from the pion $d E / d x$ peak. The integration limits were varied to check the stability of the results. The yield $Y$ can be expressed as

$$
Y=\alpha p+\beta K
$$

where $\alpha$ and $\beta$ are the proton and kaon efficiencies (fraction of Gaussian inside the integration region) from the integration described above, derived from the $d E / d x$ calibration, resolution, and the Bichsel function $[20,22]$. The kaon contamination to the proton yield is estimated by using two independent procedures. In the first case the kaon contamination is estimated through the yields of the inclusive hadrons $(h)$ and pions; in the second case using $K_{\mathrm{S}}^{0}$ measurements [21] (only available for $|y|<0.5$ up to $\left.p_{\mathrm{T}}<5 \mathrm{GeV} / \mathrm{c}\right)$. The raw proton yield is then obtained as

$$
p=(Y-\beta(h-\pi)) /(\alpha-\beta)
$$

or,

$$
p=\left(Y-\beta K_{\mathrm{S}}^{0}\right) / \alpha .
$$

The typical values of $\alpha$ for a $d E / d x$ cut slightly away from the proton peak position is 0.4 . The $\beta$ values decrease from 0.2 to 0.08 with $p_{\mathrm{T}}$ in the range $2.5<p_{\mathrm{T}}<10 \mathrm{GeV} / c$. At high $p_{\mathrm{T}}$, the yields of other stable particles (i.e., electrons and deuterons) are at least two orders of magnitude smaller than those of pions and are negligible for our studies. The two results are consistent in the region where STAR $K_{\mathrm{S}}^{0}$ measurements are available. Since the energy loss of particles in the TPC is almost independent of charge sign, the dependence of $h^{-} / h^{+}$on $n \sigma_{\pi}$ is due to different particle composition and the $d E / d x$ separation between pion, kaon and proton [20]. This provided a consistency check for the yields.

The $d E / d x$ resolution is better for longer tracks, shorter drift distance, stronger magnetic field, smaller multiplicity and lower beam luminosity. Due to longer tracks and shorter drift distances for particles produced at higher $y$, the $d E / d x$ resolution gets better. Thus, the separations between pions and kaons or (anti-)protons were larger for $0.5<|y|<1.0$ than for $|y|<0.5[21]$, and particle identification is easier at larger $p_{\mathrm{T}}$.
TABLE II: Correction factors for identified hadron spectra at high $p_{\mathrm{T}}(>2.5 \mathrm{GeV} / c)$ for minimum bias $d+\mathrm{Au}$ collisions.

\begin{tabular}{cc}
\hline Type & $\%$ \\
\hline Trigger efficiency & $95 \pm 3$ \\
Vertex efficiency & $93 \pm 1$ \\
Track reconstruction efficiency & $\sim 90 \pm 8$ \\
$(|y|<0.5)$ & \\
Track reconstruction efficiency & $\sim 82 \pm 8$ \\
$(0.5<|y|<1.0)$ & \\
Background contamination & $\sim 5 \pm 1$ \\
\hline
\end{tabular}

\section{E. Correction factors}

The various correction factors for the identified hadron spectra are listed in Table II. The trigger and vertex efficiencies were discussed previously. The identified hadron track reconstruction efficiency was estimated by embedding Monte Carlo particles into the real data and then following the full reconstruction procedure. It was observed to be independent of $p_{\mathrm{T}}$ for $p_{\mathrm{T}}>2.5 \mathrm{GeV} / \mathrm{c}$ for both rapidity regions. The reconstruction efficiency for $p_{\mathrm{T}}>2.5 \mathrm{GeV} / c$ for charged pions and protons are $\sim 92 \%$ and $\sim 90 \%$, respectively, in the rapidity region $|y|<0.5$. For $0.5<|y|<1.0$, the reconstruction efficiency for charged pions and protons is $\sim$ $82 \%$ and $84 \%$, respectively. The background contamination in the pion spectra for $p_{\mathrm{T}}>2.5 \mathrm{GeV} / c$, primarily from $K_{0}^{\mathrm{S}}$ weak decay, is $\sim 5 \%$. No strong centrality dependence was observed in the correction factors. The charged pion, proton and anti-proton spectra are corrected for efficiency and background effects. The inclusive proton and anti-proton spectra are presented without hyperon feed down corrections $[21,23]$. Preliminary study shows that the ratio of $\Lambda$ to inclusive $p$ in the rapidity range $|y|<0.5$ decreases from 0.7 to 0.3 with increase in $p_{\mathrm{T}}$ from $2.5 \mathrm{GeV} / c$ to $5.5 \mathrm{GeV} / c$.

\section{F. Systematic errors}

The total systematic uncertainties associated with the pion yields are estimated to be $\lesssim 15 \%$, and those for proton and anti-proton yields are estimated to be $\lesssim 22 \%$. They are of similar order for both the rapidity regions, and the average values for minimum bias collisions are given in Table III.

The sources of systematic error on the high $p_{\mathrm{T}}$ yield arise owing to: (a) uncertainty in modeling the detector response in the Monte Carlo simulations, (b) momentum resolution (increases with $p_{\mathrm{T}}$ ) [21], (c) difference in the yields for different TPC sectors as a result of spatial distortion effects, (d) uncertainty in determining the pion and proton $d E / d x$ 


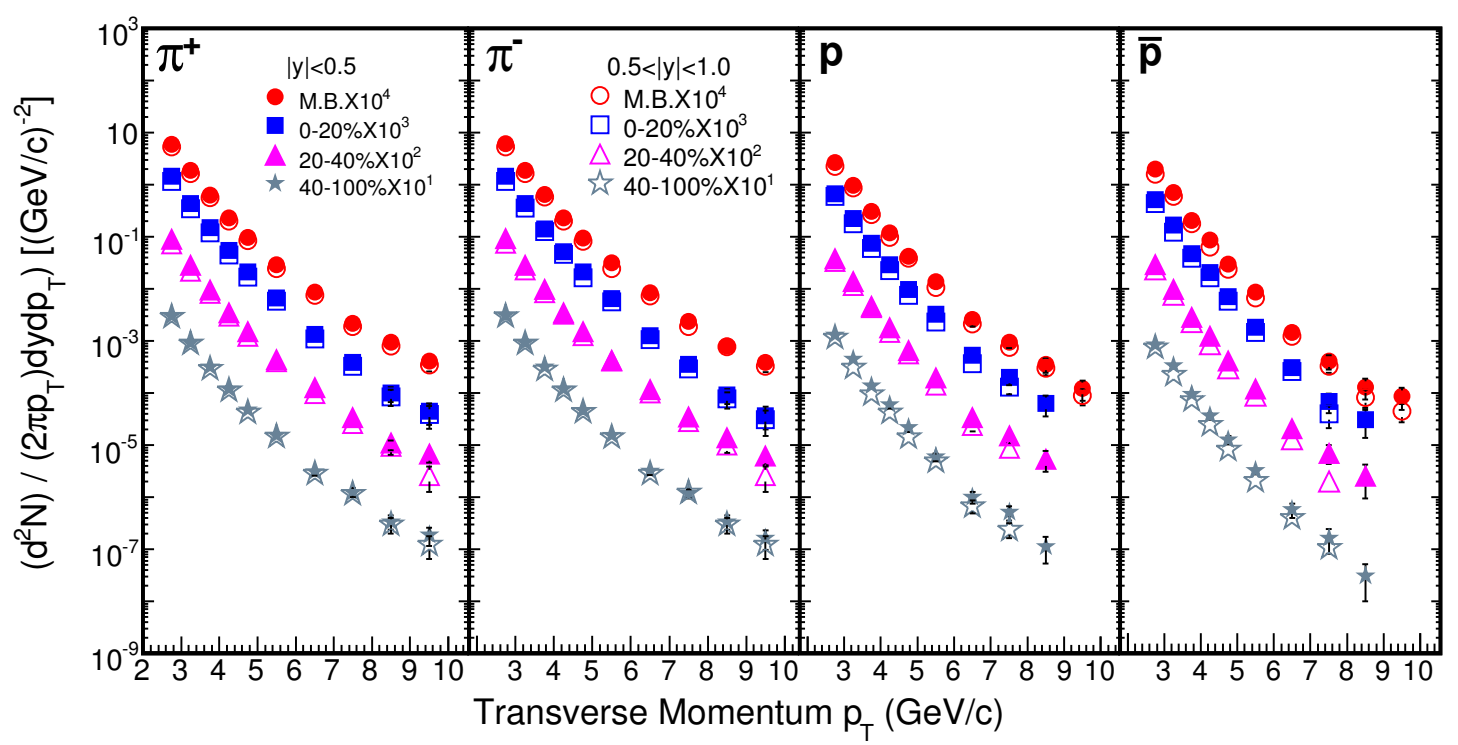

FIG. 3: (Color online) High transverse momentum spectra $\left(p_{\mathrm{T}}>2.5 \mathrm{GeV} / c\right)$ of charged pions, proton, and antiproton for the rapidity regions $|y|<0.5$ (solid symbols) and $0.5<|y|<1.0$ (open symbols) for $d+$ Au collisions and various event centrality classes at $\sqrt{s_{\mathrm{NN}}}=200 \mathrm{GeV}$.

TABLE III: Systematic errors for identified hadron minimum bias yields at high $p_{\mathrm{T}}(>2.5 \mathrm{GeV} / c)$ for $d+\mathrm{Au}$ collisions.

\begin{tabular}{cc}
\hline Sources of uncertainty & $\%$ Error \\
\hline Modeling detector response & 8 \\
Momentum resolution & $4\left(\right.$ at $\left.p_{\mathrm{T}}=7 \mathrm{GeV} / \mathrm{c}\right)$ \\
Spatial distortion & 8 \\
$d E / d x$ pion peak position & 8 \\
$d E / d x$ proton peak position & 8 \\
Kaon contamination to proton yield & $12\left(\right.$ at $\left.p_{\mathrm{T}}=7 \mathrm{GeV} / \mathrm{c}\right)$ \\
Protons from hyperon decay & $7\left(\right.$ at $\left.p_{\mathrm{T}}=7 \mathrm{GeV} / \mathrm{c}\right)$ \\
Normalization (trigger and luminosity) & 10 \\
\hline
\end{tabular}

peak positions, (e) uncertainty in estimating the kaon contamination to proton yields (increases from $7 \%$ at $p_{\mathrm{T}}=2.5 \mathrm{GeV} / c$ to $15 \%$ at $\left.p_{\mathrm{T}}=10 \mathrm{GeV} / c\right)$, and (f) uncertainty due to protons from hyperon decay that are reconstructed as primordial protons at a slightly higher $p_{\mathrm{T}}$ than their true value, with a worse momentum resolution (increases from $2 \%$ at $p_{\mathrm{T}}=2.5 \mathrm{GeV} / c$ to $10 \%$ at $\left.p_{\mathrm{T}}=10 \mathrm{GeV} / c\right)$. There is an additional $10 \%$ [15] normalization uncertainty due to trigger and luminosity uncertainties. These systematic errors are not shown in Figure 3.

As this work focuses mainly on ratios such as $Y_{\text {Asym }}$, most of the systematic errors cancel. The resultant systematic error on $Y_{\text {Asym }}$ is about $5 \%$. The errors shown for figures with ratios are statistical and systematic errors added in quadrature.

Figure 3 shows the measured invariant yields of charged pions, protons, and anti-protons for the $p_{\mathrm{T}}$ range $2.5<p_{\mathrm{T}}<10 \mathrm{GeV} / c$ in the rapidity regions, $|y|<0.5$ (solid symbols) and $0.5<|y|<1.0$ (open symbols) for minimum bias and various collision centrality classes for $d+\mathrm{Au}$ collisions at $\sqrt{s_{\mathrm{NN}}}=200 \mathrm{GeV}$. The $p_{\mathrm{T}}$ spectra are corrected for the trigger, vertex and reconstruction efficiencies and the background effects listed in Table II. The $p_{\mathrm{T}}$ bin width used in the analysis are $0.5 \mathrm{GeV} / c$ for $p_{\mathrm{T}}<5 \mathrm{GeV} / c$ and $1.0 \mathrm{GeV} / c$ for $p_{\mathrm{T}}>5 \mathrm{GeV} / c$.

\section{RAPIDITY ASYMMETRY}

In this section we discuss the $y, p_{\mathrm{T}}$, species and centrality dependence of $Y_{\text {Asym }}$.

\section{A. Rapidity, transverse momentum, and species dependence}

Figure 4 shows the high $p_{\mathrm{T}}$ dependence of $Y_{\mathrm{Asym}}$ for $\pi^{+}+\pi^{-}$and $p+\bar{p}$ for the rapidity regions $|y|<0.5$ 
and $0.5<|y|<1.0$ for minimum bias events. The backward rapidity is considered as the gold-side and corresponds to the negative rapidity region. The forward rapidity is the deuteron-side and corresponds to positive rapidity. For comparison the pseudorapidity asymmetry for charged hadrons [5] is shown also in the figure. The following observations are made:

(a) $Y_{\text {Asym }}$ is found to be larger for $0.5<|y|<1.0$ than for $|y|<0.5$ for all the hadrons with $2.5<p_{\mathrm{T}}<5 \mathrm{GeV} / c$. This may indicate the presence of some rapidity dependence of nuclear effects such as parton saturation, nuclear shadowing, or energy loss in cold nuclear matter.

(b) The $Y_{\text {Asym }}$ values are consistent with unity for both rapidity regions at high $p_{\mathrm{T}}(>5.5 \mathrm{GeV} / c)$, suggesting absence of nuclear effects on particle production in $d+\mathrm{Au}$ collisions for this $p_{\mathrm{T}}$ range. A straight line fit to the $Y_{\text {Asym }}(\pi)$ values for $p_{\mathrm{T}}>5.5 \mathrm{GeV} / c$ gives $0.99 \pm 0.04$ and $1.01 \pm 0.04$ for $|y|<0.5$ and $0.5<|y|<1.0$ respectively.

(c) $Y_{\text {Asym }}$ for charged pions is greater than unity and decreases monotonically with increasing $p_{\mathrm{T}}$ for $2.5<p_{\mathrm{T}}<5 \mathrm{GeV} / c$. Although $Y_{\mathrm{Asym}}$ for $p+\bar{p}$ is also greater than unity, the trend seems to be towards a constant value in this $p_{\mathrm{T}}$ range. These features are opposite to predictions from models based on incoherent initial multiple partonic scattering and independent fragmentation [24]. Such models predict that $Y_{\text {Asym }}$ is less than unity at intermediate $p_{\mathrm{T}}$ and approximately unity for larger $p_{\mathrm{T}}[5]$.

(d) For $|y|<0.5, Y_{\text {Asym }}$ for $p+\bar{p}$ is slightly larger than it is for charged pions for $2.5<p_{\mathrm{T}}<4 \mathrm{GeV} / c$. For $0.5<|y|<1.0$ no strong particle type dependence is observed for $Y_{\mathrm{Asym}}$. This is in contrast to the observed baryon-meson differences for the same $p_{\mathrm{T}}$ range for $\mathrm{Au}+\mathrm{Au}$ collisions, which were described by recombination-based models [25].

\section{B. Centrality dependence}

In Fig. 5 we show the centrality dependence of $Y_{\text {Asym }}$ at high $p_{\mathrm{T}}$ for $\pi^{+}+\pi^{-}$and $p+\bar{p}$ for the two rapidity regions $|y|<0.5$ (left panels) and $0.5<|y|<1.0$ (right panels). The data are shown only for $2.5<p_{\mathrm{T}}<5.5 \mathrm{GeV} / c$. The $Y_{\text {Asym }}$ values approach unity for the centrality classes studied for $p_{\mathrm{T}}>5.5 \mathrm{GeV} / c$ in both the rapidity regions. For $|y|<0.5$, a prominent centrality dependence of $Y_{\text {Asym }}$ is not observed. For $0.5<|y|<1.0, Y_{\text {Asym }}$ is larger for central (0-20\%) compared to peripheral (40-100\%) events for $2.5<p_{\mathrm{T}}<4 \mathrm{GeV} / c$. The indication of a centrality dependence in the $Y_{\mathrm{Asym}}$ at $0.5<|y|<1.0$ is consistent with predictions from saturation models [4]. However in such mod-

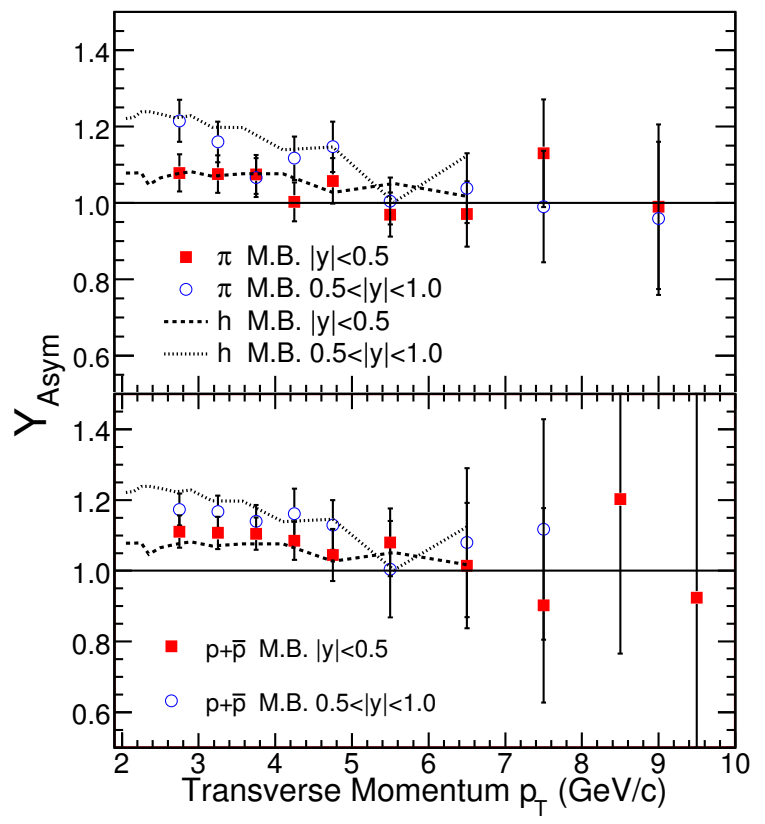

FIG. 4: (Color online) High transverse momentum rapidity asymmetry factor $\left(Y_{\mathrm{Asym}}\right)$ for $\pi^{+}+\pi^{-}$and $p+\bar{p}$ for $|y|<0.5$ and $0.5<|y|<1.0$ for minimum bias $d+\mathrm{Au}$ collisions at $\sqrt{s_{\mathrm{NN}}}=200 \mathrm{GeV}$. For comparison the inclusive charged hadron results from STAR [5] are also shown by the curves.

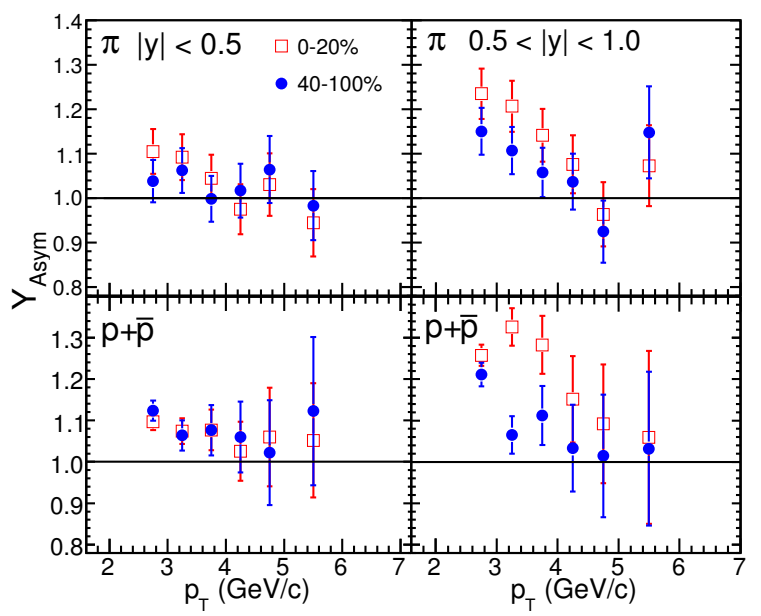

FIG. 5: (Color online) Centrality dependence of high transverse momentum rapidity asymmetry factor ( $\left.Y_{\text {Asym }}\right)$ for $\pi^{+}+\pi^{-}$and $p+\bar{p}$ at $|y|<0.5$ (left panels) and $0.5<|y|<1.0$ (right panels) for $d+\mathrm{Au}$ collisions at $\sqrt{s_{\mathrm{NN}}}=200 \mathrm{GeV}$. 
els the centrality dependence is much stronger than observed in the present data [5].

\section{MODEL COMPARISON}

In this section we compare the measured high $p_{\mathrm{T}}$ identified hadron $Y_{\text {Asym }}$ in the rapidity regions $|y|<0.5$ and $0.5<|y|<1.0$ for minimum bias $d+\mathrm{Au}$ collisions at $\sqrt{s_{\mathrm{NN}}}=200 \mathrm{GeV}$ with predictions from various models (Figs. 6-10).

\section{A. Comparison to the nuclear shadowing model}

First we compare the high $p_{\mathrm{T}}$ charged pion $Y_{\text {Asym }}$ in both rapidity regions with model predictions that incorporate only nuclear shadowing [1]. In these calculations two parameterizations of nuclear shadowing, covering the extremes of gluon shadowing at low $x$, are taken. The parametrization by Eskola et al. [26] is referred to as EKS98. The other, FGS, is from Frankfurt, Guzey, and Strikman [27] (FGSO, the original parametrization, along with FGSH and FGSL for high and low gluon shadowing). The calculations use MRST leading order (LO) parton distribution functions [28]. The fragmentation of produced partons into charged pions uses the LO Kniehl-Kramer-Potter (KKP) fragmentation functions [29] obtained from a fit to $e^{+}+e^{-}$data. In EKS98 the valence quark shadowing is identical for $u$ and $d$ quarks at the minimum momentum scale of the hard interaction. In FGS the EKS98 valence quark shadowing ratios are used as input, along with Gribov theory and hard diffraction. The charged hadron $R_{\mathrm{dAu}}$ was reasonably well-described by such a model using the FGS parametrization [1].

Our charged pion data (Fig. 6) indicate that nuclear shadowing as implemented in the models discussed, cannot explain the measured $Y_{\text {Asym }}$ for $2.5<p_{\mathrm{T}}<5 \mathrm{GeV} / c$ for both $|y|<0.5$ and $0.5<|y|<1.0$. The differences between data and model increase with increasing rapidity. At larger $p_{\mathrm{T}}$, the data values approach unity, indicating an absence of nuclear effects. The effect on $Y_{\text {Asym }}$ of using a different parametrization of nuclear shadowing at high $p_{\mathrm{T}}$ is found to be negligible for $|y|<0.5$. However, some differences are observed in FGS for $0.5<|y|<1.0$.

$Y_{\text {Asym }}$ from the nuclear shadowing model covering the extremes of gluon shadowing at low $x$ is not consistent with the measured values. The comparison therefore provides an idea of the maximum contribution to $Y_{\text {Asym }}$ from only nuclear shadowing in $d+\mathrm{Au}$ collisions at $\sqrt{s_{\mathrm{NN}}}=200 \mathrm{GeV}$.

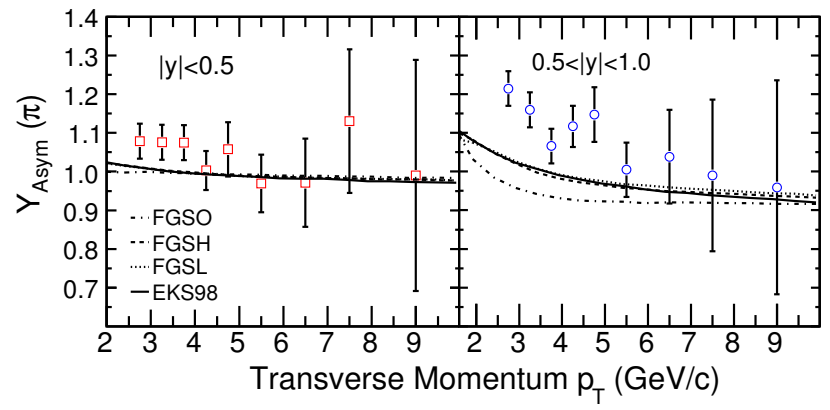

FIG. 6: (Color online) High transverse momentum rapidity asymmetry factor $\left(Y_{\mathrm{Asym}}\right)$ for $\pi^{+}+\pi^{-}$at $|y|<$ 0.5 and $0.5<|y|<1.0$ for minimum bias $d+\mathrm{Au}$ collisions at $\sqrt{s_{\mathrm{NN}}}=200 \mathrm{GeV}$ compared to a model with only nuclear shadowing [1]. The different curves represent different parametrization of nuclear shadowing. See text for more details.

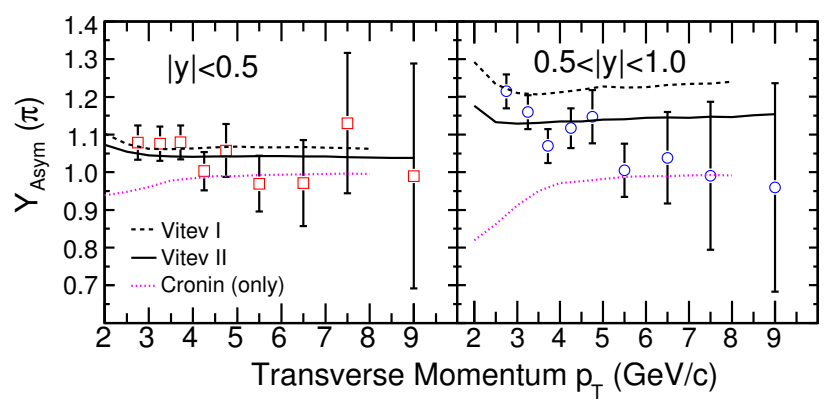

FIG. 7: (Color online) High transverse momentum rapidity asymmetry factor $\left(Y_{\mathrm{Asym}}\right)$ for $\pi^{+}+\pi^{-}$at $|y|<0.5$ and $0.5<|y|<1.0$ for minimum bias $d+\mathrm{Au}$ collisions at $\sqrt{s_{\mathrm{NN}}}=200 \mathrm{GeV}$ compared to models incorporating multiple scattering, shadowing, and energy loss in cold nuclear matter [3]. See text for more details.

\section{B. Comparison to the multiple scattering+shadowing+energy loss model}

Next we compare the high $p_{\mathrm{T}}$ charged pion $Y_{\text {Asym }}$ to a model that includes only coherent multiple scattering, which leads to transverse momentum broadening (Cronin effect), and to calculations with the addition of power corrections (dynamical shadowing) and energy loss in cold nuclear matter [3]. In this model a systematic calculation of the coherent multiple parton scattering in $p+\mathrm{A}$ collisions is carried out in terms of the perturbative QCD factorization approach. It also incorporates initial state parton energy loss in the perturbative calculations. We observe (Fig. 7) that in both rapidity regions model expectations from the Cronin effect, for $2.5<p_{\mathrm{T}}<5 \mathrm{GeV} / c$, are in qualitative 


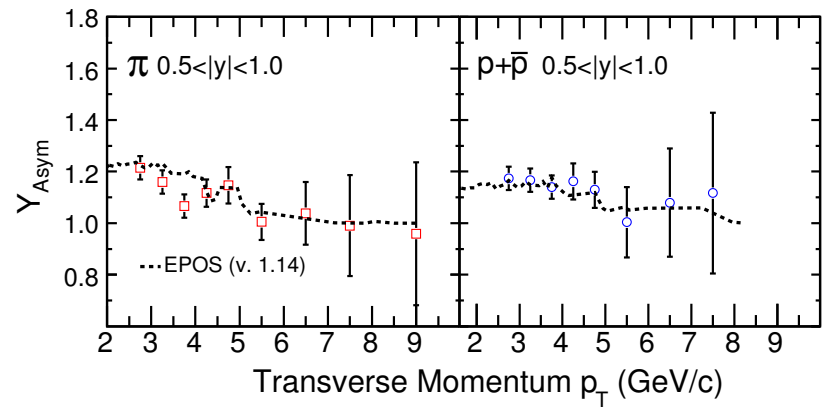

FIG. 8: (Color online) High transverse momentum rapidity asymmetry factor $\left(Y_{\mathrm{Asym}}\right)$ for $\pi^{+}+\pi^{-}$and $p+\bar{p}$ for $0.5<|y|<1.0$ for minimum bias $d+\mathrm{Au}$ collisions at $\sqrt{s_{\mathrm{NN}}}=200 \mathrm{GeV}$ compared to the EPOS model [7]. See text for more details.

disagreement with the data. This indicates that multiple scattering is not the source of the observed asymmetry. Fig. 7 shows also a comparison of the charged pion data with results of calculations which incorporate multiple scattering, dynamical shadowing, and a varying degree of energy loss in cold nuclear matter. The calculation, labeled as Vitev-I, has a slightly larger effective energy loss in cold nuclear matter than the one labeled Vitev-II. For $|y|<0.5$, both the Vitev-I and Vitev-II results are in reasonable agreement with the data within errors. For $0.5<|y|<1.0$, the Vitev-I result slightly overpredicts the measured $Y_{\text {Asym }}$. The model calculations beyond $p_{\mathrm{T}}>3 \mathrm{GeV} / c$ are independent of $p_{\mathrm{T}}$, while the measured $Y_{\text {Asym }}$ tends to decrease with $p_{\mathrm{T}}$.

For the Vitev models the rapidity dependence of $Y_{\text {Asym }}$ seems to be sensitive to effective energy loss. The decrease in $Y_{\text {Asym }}$ with $p_{\mathrm{T}}$ in the model is restricted to $p_{\mathrm{T}}<2.5 \mathrm{GeV} / c$. It will be important to have predictions from this model for proton and anti-protons to investigate the possible particle species dependence of multiple scattering in $d+\mathrm{Au}$ collisions.

\section{Comparison to the EPOS model}

In Fig. 8 the measured $Y_{\text {Asym }}$ for $\pi^{+}+\pi^{-}$and $p+\bar{p}$ for $0.5<|y|<1.0$ are compared to the results from the EPOS model. In the EPOS model [7] elastic and inelastic parton ladder splitting are the key processes. A parton ladder refers to the dynamical process of parton-parton scattering with successive emission of partons. The emission process can be an initial state, space-like cascade, or final state, timelike cascade. The elastic splitting in this model can be related to screening and saturation, while inelastic splitting is related to the hadronization process.

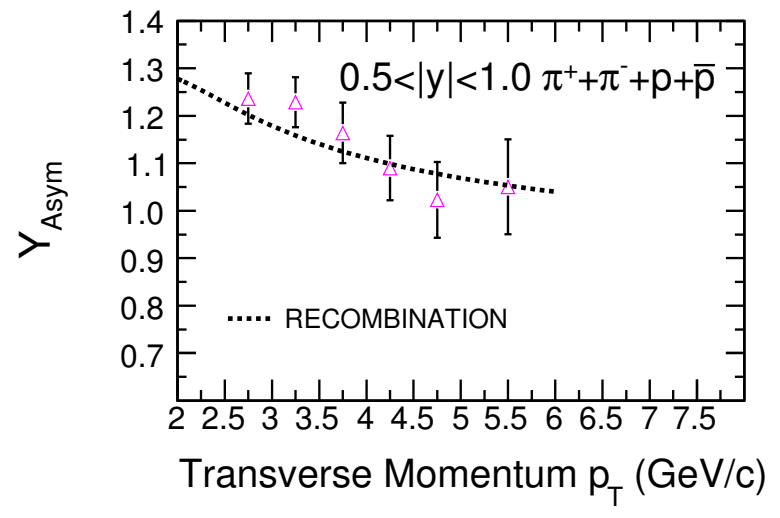

FIG. 9: (Color online) High transverse momentum rapidity asymmetry factor $\left(Y_{\mathrm{Asym}}\right)$ for $\pi^{+}+\pi^{-}+p+\bar{p}$ and 0.5 $<|y|<1.0$ in $0-20 \%$ central $d+\mathrm{Au}$ collisions at $\sqrt{s_{\mathrm{NN}}}=$ $200 \mathrm{GeV}$ compared to the recombination model [6]. See text for more details.

This phenomenological model has been very successful in describing the inclusive charged hadron $d+\mathrm{Au}$ data [7].

The EPOS model predictions (v. 1.14) are consistent with the measured $Y_{\text {Asym }}$ values for both charged pions and $p+\bar{p}$.

\section{Comparison to the recombination model}

The recombination model reproduces some of the observed features of RHIC data [6, 25]. It successfully describes the Cronin effect for $d+\mathrm{Au}$ data without any need for $k_{\mathrm{T}}$ broadening in initial state interactions. There were questions raised concerning issues such as decrease in entropy of the system and the spatial extent of the recombining subsystems. These are addressed in the Refs. [6, 25]. So it is useful to compare the experimental measurements with this model to investigate the relative importance of various physical processes.

Fig. 9 compares model predictions with the measured $Y_{\text {Asym }}$ for $\pi^{+}+\pi^{-}+p+\bar{p}$ for the rapidity region $0.5<|y|<1.0$ in $0-20 \%$ central $d+\mathrm{Au}$ collisions. The model predictions from Ref. [6] are consistent with the data. Since the pions are the dominant hadrons produced in $d+\mathrm{Au}$ collisions, the $Y_{\text {Asym }}$ for $\pi^{+}+\pi^{-}+p+\bar{p}$ is dominated by them. In the absence of predictions from this model for identified hadrons in $d+\mathrm{Au}$ data, it is not clear if it can describe the $Y_{\text {Asym }}$ for $p+\bar{p}$. One of the reasons for the success of the recombination model in the intermediate $p_{\mathrm{T}}$ range is that in this model a baryon is formed by recombination of three shower and thermal partons, while a meson needs only two, resulting in a higher 


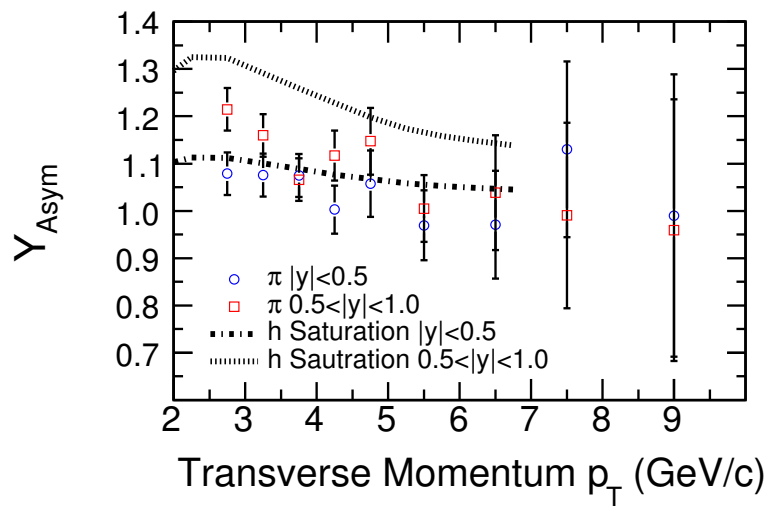

FIG. 10: (Color online) High transverse momentum rapidity asymmetry factor $\left(Y_{\text {Asym }}\right)$ for $\pi^{+}+\pi^{-}$at $|y|<0.5$ and $0.5<|y|<1.0$ for minimum bias $d+\mathrm{Au}$ collisions at $\sqrt{s_{\mathrm{NN}}}=200 \mathrm{GeV}$ compared to the saturation model [4]. See text for more details.

yield at larger momentum for baryons [6]. A comparison of model calculations separately for charged pions and $p+\bar{p}$ is of interest to see if the observed weak species dependence and almost similar $p_{\mathrm{T}}$ dependence of $Y_{\text {Asym }}$ is predicted.

\section{E. Comparison to the saturation model}

Finally, we compare our charged pion measurements to calculations from saturation models [4]. In such models the particle production is determined by the high gluon density in the $\mathrm{Au}$ nucleus and the deuteron. The model had successfully described the suppression of high $p_{\mathrm{T}}$ hadron yields at forward rapidities for $d+\mathrm{Au}$ data relative to $p+p$ data at RHIC. In contrast to a naive multiple scattering picture, where one expects enhancement due to the Cronin effect to be more significant for larger forward rapidities due to the increase in the number of scattering centers while probing smaller values of $x$, the saturation models give a completely opposite result [30]. For this model the momentum range where $Y_{\text {Asym }}>1$ is determined by the saturation and geometrical scales in the model, as well as the onset of the gluon saturation.

In Fig. 10 we compare the $Y_{\text {Asym }}$ data for charged pions with the $Y_{\text {Asym }}$ predictions for inclusive hadrons. Such a comparison is reasonable as $\pi^{+}+\pi^{-}$are the dominant hadrons produced in $d+\mathrm{Au}$ collisions. Further, the $Y_{\mathrm{Asym}}$ values for $\pi^{+}+\pi^{-}$are similar to those for $p+\bar{p}$. The model calculations are in reasonable agreement for $|y|<$ 0.5 and give the correct decreasing trend for $Y_{\mathrm{Asym}}$ vs. $p_{\mathrm{T}}$. The prediction of a strong centrality depen-

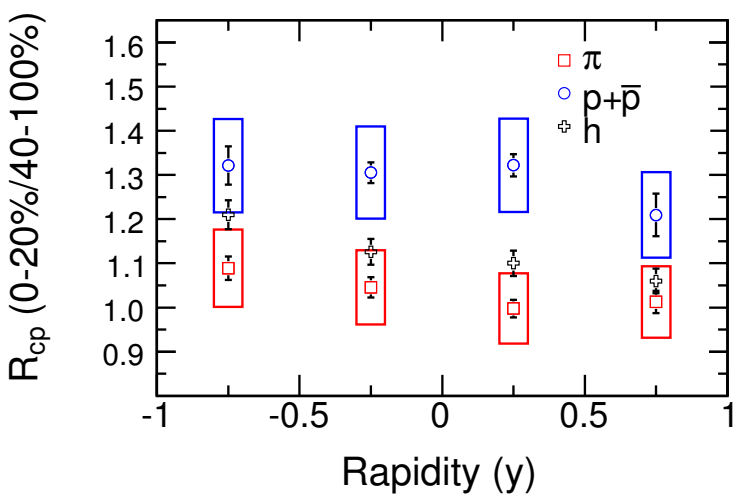

FIG. 11: (Color online) Variation of nuclear modification factor $\left(R_{\mathrm{CP}}\right)$ for $\pi^{+}+\pi^{-}$and $p+\bar{p}$ with rapidity for $p_{\mathrm{T}}>2.5 \mathrm{GeV} / c$ for $d+\mathrm{Au}$ collisions at $\sqrt{s_{\mathrm{NN}}}=200 \mathrm{GeV}$. Also shown for comparison are the $R_{\mathrm{CP}}$ values for inclusive charged hadrons as a function of pseudorapidity [5]. The errors shown as boxes are the systematic errors. The error due to number of binary collisions is $\sim 14 \%$ and is not shown in the figure.

dence at midrapidity is not observed [5]. Such models are expected to work better at forward rapidities at RHIC. The models give larger asymmetries than data for $0.5<|y|<1.0$.

In this section we compared the $Y_{\text {Asym }}$ vs. $p_{\mathrm{T}}$ to various model calculations. The $Y_{\mathrm{Asym}}$ vs. $p_{\mathrm{T}}$ dependence rules out models based on incoherent initial multiple partonic scattering and independent fragmentation. The models based only on nuclear shadowing cannot account for the measured $Y_{\text {Asym }}$. Models incorporating multiple scattering, dynamical shadowing, and energy loss in cold nuclear matter are in reasonable agreement with the data for $|y|<0.5$. However, the $Y_{\text {Asym }}$ being independent of $p_{\mathrm{T}}(>3 \mathrm{GeV} / c)$ is inconsistent with the measurements at higher rapidity. Qualitatively, features of monotonic decrease in $Y_{\text {Asym }}$ with $p_{\mathrm{T}}$ are in agreement with color-glass-condensate (CGC) type models. However, there is a lack of quantitative agreement at higher rapidities where this model is expected to work better. The EPOS and recombination models are in best quantitative agreement with the data. The actual test of the recombination model is only possible when the calculations are available for $Y_{\text {Asym }}$ for identified baryons and mesons.

\section{NUCLEAR MODIFICATION FACTOR}

The gluon saturation effects are believed to manifest themselves in terms of suppression of transverse 
distributions below the saturation scale. The onset of gluon saturation and the saturation scale, in turn, depend upon the gluon density and the rapidity of the measured particles. The saturation scale at $\mathrm{RHIC}$ is expected to be $\sim 2 \mathrm{GeV}^{2}$ and depends on the colliding nuclei and rapidity as $\sim A^{1 / 3} e^{\lambda y}$ $[4,30,31]$. The value of $\lambda$ lies between $0.2-0.3$ and is obtained from fits to HERA data [32]. It is important to study the variation of the nuclear modification factor $\left(R_{\mathrm{CP}}\right)$ as a function of rapidity. The $R_{\mathrm{CP}}(y)$ and the $Y_{\text {Asym }}\left(p_{\mathrm{T}}\right)$ together can provide a more stringent constraint on particle production models. Although the present data do not have large rapidity span, we will explore the variation of $R_{\mathrm{CP}}$ for identified hadrons from forward to backward rapidity. $R_{\mathrm{CP}}$ is defined as

$$
R_{C P}=\frac{\left.\left(d^{2} N / d p_{T} d \eta /\left\langle N_{\text {bin }}\right\rangle\right)\right|_{\text {central }}}{\left.\left(d^{2} N / d p_{T} d \eta /\left\langle N_{\text {bin }}\right\rangle\right)\right|_{\text {periph }}},
$$

where $d^{2} N / d p_{T} d \eta$ is the differential yield per event in $d+\mathrm{Au}$ collisions for a given centrality class.

Fig. 11 shows the $p_{\mathrm{T}}$ integrated $R_{\mathrm{CP}}$ for $\pi^{+}+\pi^{-}$ and $p+\bar{p}$ with rapidity $|y|<1.0$ and $p_{\mathrm{T}}>2.5$ $\mathrm{GeV} / c$. There may be a decrease in $R_{\mathrm{CP}}$ for $\pi^{+}+\pi^{-}$ from backward rapidity (gold-side) to forward rapidity (deuteron-side). Within the systematic errors (shown as boxes) the $R_{\mathrm{CP}}$ for proton+anti-proton is almost constant within the rapidity range studied. Also shown for comparison are the $R_{\mathrm{CP}}$ values for inclusive charged hadrons as a function of pseudorapidity [5]. The dependences are slightly weaker than observed by BRAHMS for inclusive charged hadrons in the forward rapidity region [33].

\section{PARTICLE RATIOS}

Figure 12 shows the $\pi^{-} / \pi^{+}$and $\bar{p} / p$ ratios for $2.5<p_{\mathrm{T}}<10 \mathrm{GeV} / c$ as functions of rapidity for peripheral (40-100\%) and $n$-tag events for $d+\mathrm{Au}$ collisions at $\sqrt{s_{\mathrm{NN}}}=200 \mathrm{GeV}$. The $\pi^{-} / \pi^{+}$ratio is unity for both $n$-tag and peripheral events in the negative (gold-side) rapidity region. For the positive rapidity region, the absolute value of $\pi^{-} / \pi^{+}$ratio is smaller for $n$-tag events compared to peripheral $d+\mathrm{Au}$ data; however considering the systematic errors (boxes), they are also consistent with unity. The systematic errors do not allow for any strong conclusions regarding the differences, which are expected from the valence quark and isospin effects at high $p_{\mathrm{T}}$ for $n$-tag events. The $\bar{p} / p$ ratios are similar within the systematic errors for the two event classes. The $\bar{p} / p$ ratios are slightly smaller than observed for $p+p$ data.

In order to cancel out most of the systematic errors (listed in Table III), we have plotted the double ratio of $\left(\pi^{-} / \pi^{+}\right)_{n-t a g} /\left(\pi^{-} / \pi^{+}\right)_{40-100 \%}$ and
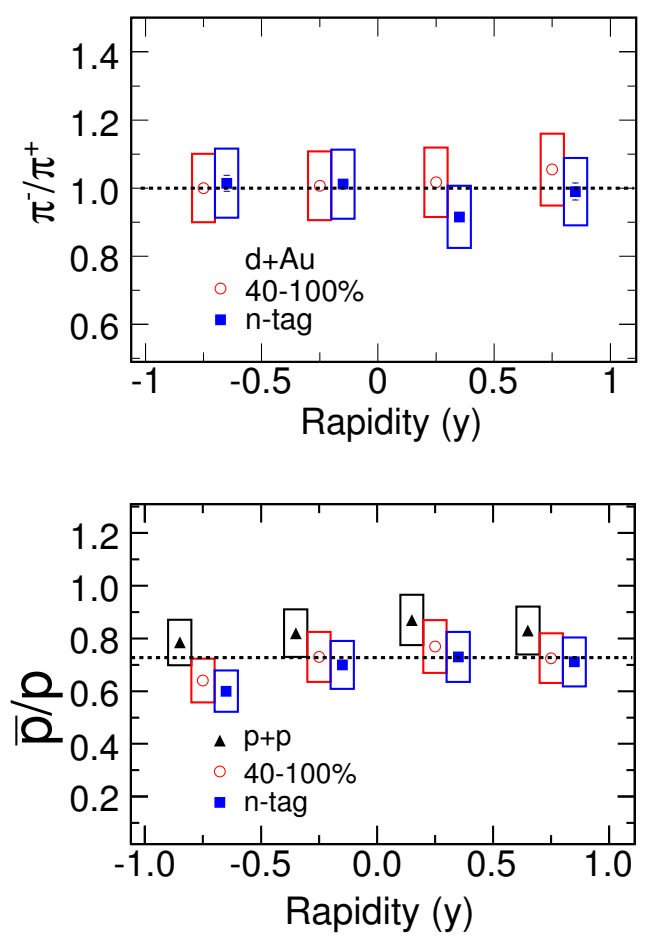

FIG. 12: (Color online) Variation of $\pi^{-} / \pi^{+}$and $\bar{p} / p$ with rapidity for $2.5<p_{\mathrm{T}}<10 \mathrm{GeV} / c$ for peripheral (40-100\%) and $n$-tag events for $d+$ Au collisions at $\sqrt{s_{\mathrm{NN}}}$ $=200 \mathrm{GeV}$. Also shown for comparison are the $\bar{p} / p$ for minimum bias $p+p$ collisions. The boxes are the systematic errors. The ratios for $n$-tag events are shifted by 0.05 units in rapidity and those for $p+p$ collisions by -0.05 units in rapidity for clarity of presentation.

$(\bar{p} / p)_{n-t a g} /(\bar{p} / p)_{40-100 \%}$ in Fig. 13. The double ratio clearly shows the difference between the $\pi^{-} / \pi^{+}$ ratio in the forward and backward rapidity regions when we compare peripheral $d+\mathrm{Au}$ collisions and $n$ tag events. A small difference for $\bar{p} / p$ ratios between $n$-tag and peripheral $d+\mathrm{Au}$ collisions is observed for both rapidities. The boxes shown in the Fig. 13 are systematic errors on the double ratio, which were calculated by varying: the distance of closest approach of the tracks from the vertex (error of $\sim 1 \%$ ), $d E / d x$ cuts (error of $\sim 1 \%$ ), $p_{\mathrm{T}}$ cuts (error of $\sim 2 \%$ ) and small change in rapidity range (error of $\sim 3 \%$ ). The total systematic error on the double ratio is $\sim$ $4 \%$.

The particle ratios and the double ratios can be used to get an idea of relative fragmentation of $d$ and $u$ quarks to protons, as well as $u$-quarks to $\pi^{+}$and $\pi^{-}$. The details of the procedure relating the measured ratios to the fragmentation ratios are given in Ref. [9]. Below we follow a similar procedure (and notations) to relate our measurements of $\pi^{-} / \pi^{+}$double ratio to the underlying quark and 


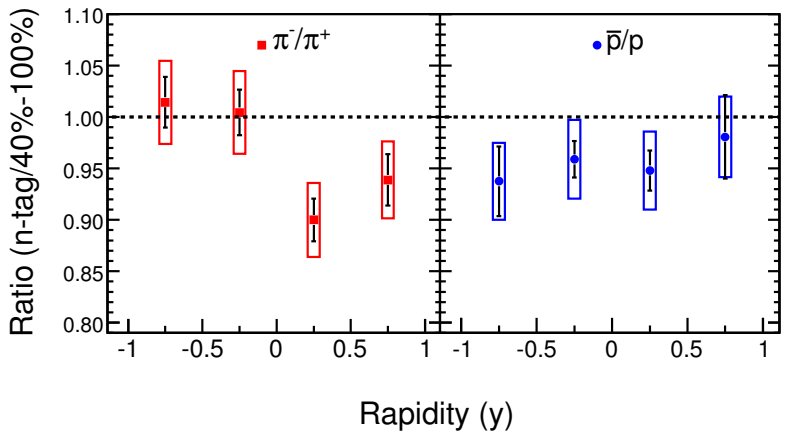

FIG. 13: (Color online) Variation of double ratio $\left(\pi^{-} / \pi^{+}\right)_{n-\text { tag }} /\left(\pi^{-} / \pi^{+}\right)_{40-100 \%}$ and $(\bar{p} / p)_{n-t a g} /(\bar{p} / p)_{40-100 \%}$ with rapidity for $2.5<p_{\mathrm{T}}<10$ $\mathrm{GeV} / c$ for $d+\mathrm{Au}$ collisions at $\sqrt{s_{\mathrm{NN}}}=200 \mathrm{GeV}$. The boxes are the systematic errors.

gluon contributions to pion production at high $p_{\mathrm{T}}$. We assume (as in Ref. [9]) charge conjugation invariance, isospin rotation symmetry for the quark fragmentation functions and NLO pQCD gluon and valence quark contribution only. The gluons are considered to fragment equally to $\pi^{+}$and $\pi^{-}$at high $p_{\mathrm{T}}$. In addition, the $\pi^{-} / \pi^{+}$double ratios at backward rapidity $(y<0)$ which is consistent with unity, suggest that the contribution of quark fragmentation from forward projectile (deuteron) is very small. This, in turn indicates that the quark contribution from $\mathrm{Au}$ side to the pion production at forward rapidity is small. In the following derivations we neglect the quark contribution from the Au side to the forward pion production as suggested by the data.

The $\pi^{+}$production for $y>0$ in $n$-tagged $d+\mathrm{Au}$ events (effective $p+A u$ collisions) is given as

$$
A+2 f_{q} D_{u}^{\pi^{+}}+f_{q} D_{q}^{\pi^{-}}
$$

and similarly for $\pi^{-}$as,

$$
A+2 f_{q} D_{u}^{\pi^{-}}+f_{q} D_{q}^{\pi^{+}} .
$$

Where $\mathrm{A}$ is the gluon contribution, $f_{q}$ is the single valence quark contribution and $D_{u}^{\pi}$ is the quark fragmentation to pion in the $p_{\mathrm{T}}$ region studied. Isospin rotation symmetry which leads to $D_{u}^{\pi^{+}}=D_{q}^{\pi^{-}}$and $D_{u}^{\pi^{-}}=D_{q}^{\pi^{+}}$is used to arrive at the above expressions.

If $x_{q}^{\pi}$ is the quark contribution to pion production in peripheral $d+\mathrm{Au}$ collisions, then it can be shown

$$
A=\frac{\left(1-x_{q}^{\pi}\right)}{x_{q}^{\pi}} 1.5 f_{q}\left(D_{q}^{\pi^{+}}+D_{q}^{\pi^{-}}\right)
$$

As mentioned above, we assume no quark contribution from $\mathrm{Au}$ nucleus at forward rapidity as the ratio of $\pi^{-} / \pi^{+}$is unity for peripheral $d+\mathrm{Au}$ collisions. Then the double ratio is given as

$$
r_{\pi}=\frac{A+2 f_{q} D_{u}^{\pi^{+}}+f_{q} D_{q}^{\pi^{-}}}{A+2 f_{q} D_{u}^{\pi^{-}}+f_{q} D_{q}^{\pi^{+}}} .
$$

Substituting the value of A from previous expression we get, ratio of $u$-quarks fragmenting to $\pi^{-}$ $\left(D_{u}^{\pi^{-}}\right)$to $u$-quarks fragmenting to $\pi^{+}\left(D_{u}^{\pi^{+}}\right)$which is given as

$$
\frac{D_{q}^{\pi^{-}}}{D_{u}^{\pi^{+}}}=\frac{1-\frac{1-r_{\pi}}{x_{q}^{\pi}}\left(1.5+0.5 x_{q}^{\pi}\right)}{1-\frac{1-r_{\pi}}{x_{q}^{\pi}}\left(1.5-0.5 x_{q}^{\pi}\right)} .
$$

Figure 14 shows the ratio $\frac{D_{q}^{\pi^{-}}}{D_{u}^{\pi^{+}}}$as a function of $x_{q}^{\pi}$ for the measured double ratio $r_{\pi}$ as given in Fig. 13 . The dashed lines reflect the $1 \sigma$ uncertainty in the ratio $\frac{D_{q}^{\pi^{-}}}{D_{u}^{\pi^{+}}}$due to uncertainty in the measurements of the double ratio of $\pi^{-} / \pi^{+}$or $r_{\pi}$. The horizontal shaded band reflects the $x_{q}^{\pi}$ value for charged pions from NLO pQCD calculations using the AlbinoKniehl-Kramer (AKK) set of fragmentation functions (FFs) [34]. The width of this band reflects the uncertainty associated with $x_{q}^{\pi}$ from the NLO pQCD calculations. These are obtained by varying the factorization scales from $0.5 p_{\mathrm{T}}$ to $2 p_{\mathrm{T}}$. Since the NLO pQCD calculations with AKK FFs agree reasonably well with charged pion measurements at RHIC [21], from the figure we can conclude that for the $\frac{{D_{q}^{\pi^{-}}}^{D_{u}^{+}}}{D_{u}^{+}}=$ 0.3 to 0.6 , our measurement is consistent with $x_{q}^{\pi}=$ $18 \%$ when compared to NLO pQCD caluclations.

Similarly, it can be shown that the ratio of $u$-quark fragmenting to protons $\left(D_{u}^{p}\right)$, to $d$-quark fragmenting to protons $\left(D_{d}^{p}\right)$ is given as,

$$
\frac{D_{u}^{p}}{D_{d}^{p}}=1+6 \frac{\left(1-r_{p 2}\right)}{\left(4 r_{p 2}-r_{p 1} r_{p 2}-3\right)},
$$

where $r_{p 1}$ is the ratio $\bar{p} / p$ in $n$-tag events, and $r_{p 2}$ is the double ratio of $\bar{p} / p$ shown in Fig. 13. Similar measurements have been carried out by the OPAL collaboration for $e^{+}+e^{-}$collisions as a function of $x_{p}$ $\left(2 \mathrm{p} / \sqrt{s}\right.$, largest scaled momentum) [10] for $x_{p}>0.5$. The values are consistent with our current measurements of the double ratios with large uncertainties in these measurements. We also have to assume that all the anti-protons at high $p_{\mathrm{T}}$ are from gluon fragmentation and that gluons fragment equally to protons and anti-protons. Only valence quarks contribute to the proton production at forward rapidity and viceversa. Our data in Fig. 13 right panel seem to show flat distribution in $-1<y<1$ and therefore may invalidate this assumption. 


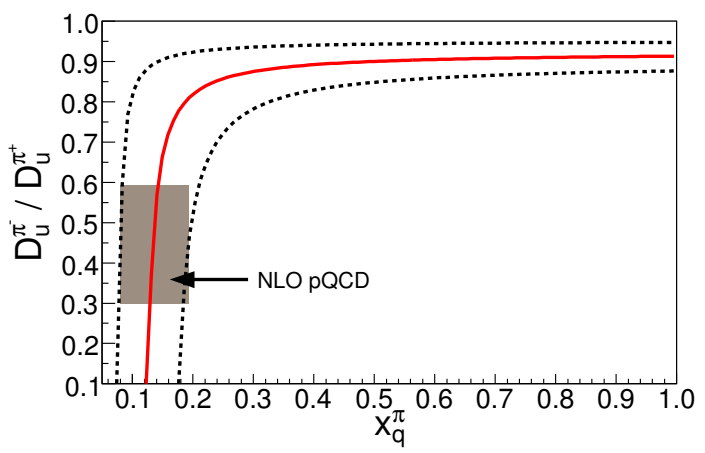

FIG. 14: (Color online) Variation of $\frac{D_{q}^{\pi^{-}}}{D_{u}^{\pi^{+}}}$with fraction of pions $\left(x_{q}^{\pi}\right)$ originating from quark jets (solid line) obtained from the measured ratios of $\pi^{-} / \pi^{+}$in peripheral $d+\mathrm{Au}$ collisions and $n$-tag events. The dashed lines reflects $1 \sigma$ systematic errors in obtaining the $\frac{D_{q}^{\pi^{-}}}{D_{u}^{\pi^{+}}}$. The horizontal shaded band reflects the possible $x_{q}^{\pi}$ range obtained from NLO pQCD calculations using AKK fragmentation functions [34].

\section{SUMMARY}

We have presented transverse momentum spectra for identified charged pions, protons and antiprotons from $d+\mathrm{Au}$ collisions in various centrality classes at $\sqrt{s_{\mathrm{NN}}}=200 \mathrm{GeV}$. The transverse momentum spectra are measured in 4 rapidity bins for $-1<y<1$ over the range $2.5<p_{\mathrm{T}}<10 \mathrm{GeV} / c$. The rapidity, $p_{\mathrm{T}}$, centrality, and species dependence of the rapidity asymmetry $Y_{\text {Asym }}$ has been studied. We have also presented the rapidity dependence of the nuclear modification factor and the $\pi^{-} / \pi^{+}$ and $\bar{p} / p$ ratios for the rapidity range $|y|<1.0$ and $p_{\mathrm{T}}>2.5 \mathrm{GeV} / c$.

The $Y_{\text {Asym }}$ is found to be larger for $0.5<|y|<1.0$ than for $|y|<0.5$ in the range $2.5<p_{\mathrm{T}}<5.0 \mathrm{GeV} / c$. For higher $p_{\mathrm{T}}$ the $Y_{\text {Asym }}$ approach 1 for both charged pions and $p+\bar{p}$. From these observations we conclude that possible sources of nuclear effects in $d+$ Au collisions, such as parton saturation, nuclear shadowing, or energy loss in cold nuclear matter, have a strong rapidity dependence which vanishes for $p_{\mathrm{T}}>5.5$ $\mathrm{GeV} / c$. The observed $Y_{\text {Asym }}$ vs. $p_{\mathrm{T}}$ dependence rules out models based on incoherent initial multiple partonic scattering and independent fragmentation.

Comparison to models based on nuclear shadowing reveals that incorporation of extremes of gluon shadowing at low $x$ does not reproduce the measured $Y_{\text {Asym }}$. This provides an upper limit on the contribution of nuclear shadowing to the $Y_{\text {Asym. }}$. Models incorporating multiple scattering, dynamical shadowing, and energy loss in cold nuclear matter are in reasonable agreement with the data for $|y|<$ 0.5. However, the $Y_{\text {Asym }}$ being independent of $p_{\mathrm{T}}$ $(>3 \mathrm{GeV} / c)$ is inconsistent with the measurements at higher rapidity. Qualitatively, features of monotonic decrease in $Y_{\text {Asym }}$ with $p_{\mathrm{T}}$ and $R_{\mathrm{CP}}$ with $y$ are in agreement with color-glass-condensate (CGC) type models. However, there is a lack of quantitative agreement at higher rapidities where this model is expected to work better. Further, the absence of very strong centrality dependence at midrapidity in the data is in contrast to the predictions from CGC models.

The EPOS and recombination models are in best quantitative agreement with the data. The actual test of the recombination model is only possible when the calculations are available for $Y_{\text {Asym }}$ for identified baryons and mesons. It will be interesting to see if this model can explain the observed weak species dependence and similar $p_{\mathrm{T}}$ dependence of $Y_{\mathrm{Asym}}$ for $\pi^{+}+\pi^{-}$and $p+\bar{p}$.

In general, the study of identified hadron $Y_{\text {Asym }}$ as a function of many variables $\left(y, p_{\mathrm{T}}\right.$, centrality and particle type) for $d+\mathrm{Au}$ collisions has been able to provide some definitive insight on mechanisms of particle production in $d+\mathrm{Au}$ collisions at $\sqrt{s_{\mathrm{NN}}}=$ $200 \mathrm{GeV}$. The $Y_{\mathrm{Asym}}\left(p_{\mathrm{T}}\right)$ together with $R_{\mathrm{CP}}(y)$ can provide a more stringent constrain on particle production models. It may be mentioned that a detailed of study of particle yields $\left(p_{\mathrm{T}}<3 \mathrm{GeV} / c\right)$ at midrapidity and forward rapidity in STAR has revealed a possible alternative explanation of the pseudorapidity dependence of $R_{\mathrm{CP}}$ from a purely geometrical picture. The decrease in $R_{\mathrm{CP}}$ from negative (backward) to positive (forward) rapidity can be explained by considering the initial asymmetry in particle production in $d+\mathrm{Au}$ collisions compared to the symmetric $p+p$ collisions [35].

The ratios $\pi^{-} / \pi^{+}$and $\bar{p} / p$ have been studied for peripheral and $n$-tag events for $d+$ Au collisions to see the possible valence quark effect. For the range $2.5<p_{\mathrm{T}}<10 \mathrm{GeV} / c$ and the rapidity region on the deuteron-side, the ratios for $n$-tag events are smaller than for peripheral events. However, within the systematic errors it is difficult to make strong conclusions of valence quark effects on particle production at high $p_{\mathrm{T}}$. The $\bar{p} / p$ ratios are observed to be systematically lower than corresponding values from $p+p$ collisions.

The double ratio between $n$-tag events and 40$100 \%$ peripheral collision events does reveal a clear enhancement in $\pi^{+}$production relative to $\pi^{-}$at forward rapidity (deuteron-side). No such enhancement is observed at the backward rapidity (goldside). Using the above ratio measurements we have found for $\frac{D_{q}^{\pi^{-}}}{D_{u}^{\pi^{+}}}=0.3$ to 0.6 , our measurement is 
consistent with $x_{q}^{\pi}=18 \%$ when compared to NLO pQCD caluclations.

A future, high statistics run for $d+\mathrm{Au}$ collisions at RHIC may be able to provide data that will lead to a still better insight into valence quark and gluon contribution, as well as isospin effects at high $p_{\mathrm{T}}$.

\section{Acknowledgments}

We would like to thank S. Albino, R. Hwa, I. Vitev, R. Vogt, K. Werner, and C. B. Yang for providing us the results for the different model calculations and many useful discussions. We thank the
RHIC Operations Group and RCF at BNL, and the NERSC Center at LBNL for their support. This work was supported in part by the Offices of NP and HEP within the U.S. DOE Office of Science; the U.S. NSF; the BMBF of Germany; CNRS/IN2P3, RA, RPL, and EMN of France; EPSRC of the United Kingdom; FAPESP of Brazil; the Russian Ministry of Science and Technology; the Ministry of Education and the NNSFC of China; IRP and GA of the Czech Republic, FOM of the Netherlands, DAE, DST, and CSIR of the Government of India; Swiss NSF; the Polish State Committee for Scientific Research; SRDA of Slovakia, and the Korea Sci. \& Eng. Foundation.
[1] R. Vogt, Phys. Rev. C 70, 064902 (2004).

[2] D. Antreasyan et al., Phys. Rev. D 19, 764 (1979).

[3] J. Qiu and I. Vitev, Phys. Lett. B 632, 507 (2006); I. Vitev, Phys. Lett. B 562, 36 (2003).

[4] D. Kharzeev, Y. Kovchegov and K. Tuchin, Phys. Lett. B 599, 23 (2004).

[5] STAR Collaboration, J. Adams et al., Phys. Rev. C 70, 064907 (2004).

[6] R. Hwa, C.B. Yang and R. J. Fries, Phys. Rev. C 71, 024902 (2005).

[7] K. Werner, F. Liu and T. Pierog, arXiv:hep$\mathrm{ph} / 0506232$.

[8] STAR Collaboration, J. Adams et al., Phys. Rev. Lett. 92, 052302 (2004).

[9] European Muon Collaboration, J. J. Aubert et al., Phys. Lett. B 160, 417 (1985); M. Arneodo et al., Nucl. Phys. B 321, 541 (1989).

[10] OPAL Collaboration, G. Abbiendi et al., Eur. Phys. J. C 16, 407 (2000).

[11] K. H. Ackerman et al., Nucl. Instrum. Methods A 499, 624 (2003).

[12] M. Anderson et al., Nucl. Instrum. Methods A 499, 659 (2003).

[13] C. Adler et al., Nucl. Instrum. Methods A 499, 433 (2003); Nucl. Instrum. Methods A 461, 337 (2001).

[14] K. H. Ackermann et al., Nucl. Instrum. Methods A 499, 713 (2003).

[15] STAR Collaboration, J. Adams et al., Phys. Rev. Lett. 91, 072304 (2003).

[16] STAR Collaboration, C. Adler et al., Phys. Rev. Lett. 89, 202301 (2002); B. Choi, Ph.D. Dissertation, University of Texas (2003).

[17] D. Kharzeev, E. Levin and M. Nardi, Nucl. Phys. A 730, 448 (2004); L. Hulthén and M. Sagawara, Handbuch der Physik (Springer-Verlag, Berlin, 1957), Vol. 39.

[18] R.E. Ansorge et al., Z. Phys. C 43, 357 (1989).

[19] X.N. Wang and M. Gyulassy, Phys. Rev. D 44, 3501 (1991). Version 1.382 is used.

[20] M. Shao et al., Nucl. Instrum. Methods A 558, 419 (2006).

[21] STAR Collaboration, J. Adams et al., Phys. Lett. B
637, 161 (2006).

[22] H. Bichsel, Nucl. Instrum. Methods A 562, 154 (2006); H. Bichsel, D. E. Groom, S. R. Klein, Phys. Lett. B 592, 242 (2004); Proceedings of 8th International Conference on Advance Technology and Particle Physics, ICATPP 2003, p. 448.

[23] STAR Collaboration, J. Adams et al., Phys. Lett. B 616, 8 (2005).

[24] X-N. Wang, Phys. Lett. B 565, 116 (2003).

[25] V. Greco, C.M. Ko and P. Levai, Phys. Rev. Lett. 90, 202302 (2003); Phys. Rev. C 68, 034904 (2003). R. J. Fries, B. Muller, C. Nonaka and S. A. Bass, Phys. Rev. Lett. 90, 202303 (2003); Phys. Rev. C 68, 044902 (2003).

[26] K. J. Eskola, V. J. Kolhinen, and P. V. Ruuskanen, Nucl. Phys. B 535, 351 (1998); K. J. Eskola, V. J. Kolhinen, and C. A. Salgado, Eur. Phys. J. C 9, 61 (1999).

[27] L. Frankfurt, V. Guzey, and M. Strikman, Phys. Rev. D 71, 054001 (2005).

[28] A. D. Martin, R.G. Roberts and W. J. Stirling, Phys. Lett. B 354, 155 (1995).

[29] B. A. Kniehl, G. Kramer and B. Potter, Nucl. Phys. B 597, 337 (2001).

[30] D. Kharzeev, Y. Kovchegov and K. Tuchin, Phys. Rev. D 68, 094013 (2003).

[31] L. McLerran and R. Venugopalan, Phys. Rev. D 49, 2233 (1994); Phys. Rev. D 59, 094002 (1999); E. Iancu, A. Leoidov and L. D. McLerran, Nucl. Phys. A 692, 583 (2001); A. Dumitru and J. JalilianMarian, Phys. Lett. B 547, 15 (2002); F. Gelis and J. Jalilian-Marian, Phys. Rev. D 66, 014021 (2002).

[32] ZEUS Collaboration, J. Breitweg et al., Phys. Lett. B 407, 432 (1997).

[33] BRAHMS Collaboration, I. Arsene et al., Phys. Rev. Lett. 93, 242303 (2004).

[34] S. Albino, B. A. Kniehl and G. Kramer, Nucl. Phys. B 725, 181 (2005).

[35] J. Putschke ( for the STAR Collaboration), J. Phys. Conf. Ser. 5, 37 (2005). 